\title{
ON THE GLOBAL WELL-POSEDNESS OF 3-D AXI-SYMMETRIC NAVIER-STOKES SYSTEM WITH SMALL SWIRL COMPONENT
}

\author{
YANLIN LIU AND PING ZHANG
}

\begin{abstract}
In this paper, we prove the local well-posedness of 3-D axi-symmetric NavierStokes system with initial data in the critical Lebesgue spaces. We also obtain the global well-posedness result with small initial data. Furthermore, with the initial swirl component of the velocity being sufficiently small in the almost critical spaces, we can still prove the global well-posedness of the system.
\end{abstract}

Keywords: Axi-symmetric Navier-Stokes system, critical spaces, mild solution.

\section{INTRODUCTION}

In this paper, we investigate the well-posedness and long-time behavior of global solutions to 3D axisymmetric Navier-Stokes equations with a small swirl component. In general, 3-D Navier-Stokes system in $\mathbb{R}^{3}$ reads

$$
\left\{\begin{array}{l}
\partial_{t} u+u \cdot \nabla u-\Delta u+\nabla p=0, \quad(t, x) \in \mathbb{R}^{+} \times \mathbb{R}^{3} \\
\operatorname{div} u=0 \\
\left.u\right|_{t=0}=u_{0},
\end{array}\right.
$$

where $u(t, x)=\left(u^{1}, u^{2}, u^{3}\right)$ stands for the velocity field and $p$ the scalar pressure function of the fluid, which guarantees the divergence free condition of the velocity field. This system describes the motion of viscous incompressible fluid flows.

We recall that except the initial data with special structure, it is not known whether or not the system (1.1) has a unique global smooth solution with large smooth initial data. For instance, the system (1.1) is globally well-posed for data which is axisymmetric and without swirl component (that is the case when $u^{\theta}=0$ in (1.3) below). In this case, Ladyzhenskaya [7] and independently Ukhovskii and Yudovich [11] proved the existence of weak solutions along with the uniqueness and regularities of such solution for (1.1). Leonardi, Málek, Nečas and Pokorny [8] gave a refined proof of the same result in [7, 11]. And even with a small swirl component, the authors [12] could also establish the global well-posedess of (1.1). In general, even the global wellposedness of (1.1) with axisymmetric initial data is still open.

On the other hand, in the seminal paper [10], Leray proved the global existence of finite energy weak solutions to (1.1). Yet the uniqueness and regularity to this weak solution are big open questions in the field of mathematical fluid mechanics. Furthermore, Leray emphasized two facts about Navier-Stokes system. Firstly, he pointed out that energy estimate method is very important to study navier-Stokes system. The general energy inequality for (1.1)

$$
\frac{1}{2}\|v(t)\|_{L^{2}}^{2}+\int_{0}^{t}\left\|\nabla v\left(t^{\prime}\right)\right\|_{L^{2}}^{2} d t^{\prime}=\frac{1}{2}\left\|v_{0}\right\|_{L^{2}}^{2},
$$

is the cornerstone of the proof to the existence of global turbulent solution to (1.1) in [10]. The energy estimate relies (formally) on the fact that if $v$ is a divergence free vector field, $(v$.

Date: September 7, 2018. 
$\nabla f \mid f)_{L^{2}}=0$ and that $(\nabla p \mid v)_{L^{2}}=0$. In the present work, we shall use the more general fact that for any divergence free vector field $v$ and any function $a$, we have

$$
\left.\int_{\mathbb{R}^{3}} v(x) \cdot \nabla a(x)|a(x)|^{p-2} a(x) d x=0 \quad \text { for any } p \in\right] 1, \infty[.
$$

This will lead to the $L^{p}$ type energy estimate. Secondly Leray pointed out that the scaling invariance of (1.1), that is,

$$
v(t, x) \mapsto \lambda v\left(\lambda^{2} t, \lambda x\right) \text { and } \quad p(t, x) \mapsto \lambda^{2} p\left(\left(\lambda^{2} t, \lambda x\right),\right.
$$

if $(v, p)$ is a solution of $(1.1)$ on $[0, T] \times \mathbb{R}^{3}$ associated with an initial data $v_{0}$, then $\left(v_{\lambda}, p_{\lambda}\right)$ is also a solution of $(1.1)$ on $\left[0, \lambda^{-2} T\right] \times \mathbb{R}^{3}$ associated with the initial data $\lambda v_{0}(\lambda x)$, is another important fact in the study of Navier-Stokes system. The scaling property is also the foundation of the Kato theory which gives a general method to solve (locally or globally) the incompressible Navier-Stokes equation in critical spaces i.e. spaces with the norms of which are invariant under the scaling. In what follows, we shall use such scaling invariant space as $L^{\infty}(] 0, t\left[; L^{1}(\Omega)\right)$, where the norm $L^{1}(\Omega)$ is given by $(1.5)$.

In fact, Gally and $\breve{S}$ verák [5] recently proved the global well-posedness of 3-D axisymmetric Navier-Stokes system without swirl and with initial data in the scaling invariant function spaces. We remark that the reason why one can prove the global well-posedness of (1.1) in this case is due to the $\theta$ component of the vorticity, $\omega^{\theta}$, satisfies

$$
\partial_{t} \frac{\omega^{\theta}}{r}+\left(u^{r} \partial_{r}+u^{z} \partial_{z}\right) \frac{\omega^{\theta}}{r}-\left(\Delta+\frac{2}{r} \partial_{r}\right) \frac{\omega^{\theta}}{r}=0 .
$$

The scaling invariant Lebesgue space for $\frac{\omega^{\theta}}{r}$ is $L^{\infty}(] 0, t\left[; L^{1}\left(\mathbb{R}^{3}\right)\right)$. Motivated by [5], the purpose of this paper is to improve the norm for the initial data in [12] to be scaling invariant ones. We remark that the other motivation of this paper comes from [3] where the authors proved that one scaling invariant norm to one component of Navier-Stokes system controls the regularity of the solution. Yet we still do not know in general the global well-posedness of Naver-Stokes with one component being small in some scaling invariant space.

Now we restrict ourselves to the axisymmetric solutions of (1.1) with the following form

$$
u(t, x)=u^{r}(t, r, z) e_{r}+u^{\theta}(t, r, z) e_{\theta}+u^{z}(t, r, z) e_{z},
$$

where $(r, \theta, z)$ denotes the usual cylindrical coordinates in $\mathbb{R}^{3}$ so that $x=(r \cos \theta, r \sin \theta, z)$, and

$$
e_{r}=(\cos \theta, \sin \theta, 0), e_{\theta}=(-\sin \theta, \cos \theta, 0), e_{z}=(0,0,1), r=\sqrt{x_{1}^{2}+x_{2}^{2}} .
$$

Then in this case, we can reformulate (1.1) as

$$
\left\{\begin{array}{l}
\partial_{t} u^{r}+\left(u^{r} \partial_{r}+u^{z} \partial_{z}\right) u^{r}-\left(\partial_{r}^{2}+\partial_{z}^{2}+\frac{1}{r} \partial_{r}-\frac{1}{r^{2}}\right) u^{r}-\frac{\left(u^{\theta}\right)^{2}}{r}+\partial_{r} p=0 \\
\partial_{t} u^{\theta}+\left(u^{r} \partial_{r}+u^{z} \partial_{z}\right) u^{\theta}-\left(\partial_{r}^{2}+\partial_{z}^{2}+\frac{1}{r} \partial_{r}-\frac{1}{r^{2}}\right) u^{\theta}+\frac{u^{r} u^{\theta}}{r}=0 \\
\partial_{t} u^{z}+\left(u^{r} \partial_{r}+u^{z} \partial_{z}\right) u^{z}-\left(\partial_{r}^{2}+\partial_{z}^{2}+\frac{1}{r} \partial_{r}\right) u^{z}+\partial_{z} p=0 \\
\partial_{r} u^{r}+\frac{1}{r} u^{r}+\partial_{z} u^{z}=0 \\
\left.u\right|_{t=0}=u_{0}
\end{array}\right.
$$

Let us denote $\widetilde{u} \stackrel{\text { def }}{=} u^{r} e_{r}+u^{z} e_{z}$. Then it is easy to check that

$$
\operatorname{div} \widetilde{u}=0 \text { and } \operatorname{curl} \widetilde{u}=\omega^{\theta} e_{\theta},
$$


so that the Biot-Savart law shows that $u^{r}$ and $u^{z}$ can be uniquely determined by $\omega^{\theta}$ (see Subsection 2.1). Hence we can write the System (1.3) as

$$
\left\{\begin{array}{l}
\partial_{t} \omega^{\theta}-\left(\partial_{r}^{2}+\partial_{z}^{2}+\frac{1}{r} \partial_{r}-\frac{1}{r^{2}}\right) \omega^{\theta}=-\operatorname{div}_{*}\left(\widetilde{u} \omega^{\theta}\right)+\frac{2 u^{\theta} \partial_{z} u^{\theta}}{r} \\
\partial_{t} u^{\theta}-\left(\partial_{r}^{2}+\partial_{z}^{2}+\frac{1}{r} \partial_{r}-\frac{1}{r^{2}}\right) u^{\theta}=-\operatorname{div}_{*}\left(\widetilde{u} u^{\theta}\right)-\frac{2 u^{\theta} u^{r}}{r} \\
\left.\left(\omega^{\theta}, u^{\theta}\right)\right|_{t=0}=\left(\omega_{0}^{\theta}, u_{0}^{\theta}\right) .
\end{array}\right.
$$

Here and all in that follows, we always denote $\operatorname{div}_{*} f \stackrel{\text { def }}{=} \partial_{r} f^{r}+\partial_{z} f^{z}$ and abuse the notation $\tilde{u}=\left(u^{r}, u^{z}\right)$.

As in [5], we shall equip the half-plane $\Omega=\{(r, z) \mid r>0, z \in \mathbb{R}\}$ with the 2D measure $d r d z$, instead of the 3D measure $r d r d z$. For any $p \in\left[1, \infty\left[\right.\right.$, we denote by $L^{p}(\Omega)$ the space of measurable functions $f: \Omega \rightarrow \mathbb{R}$ which verifies

$$
\|f\|_{L^{p}(\Omega)} \stackrel{\text { def }}{=}\left(\int_{\Omega}|f(r, z)|^{p} d r d z\right)^{\frac{1}{p}}<\infty, \quad 1 \leq p<\infty .
$$

The space $L^{\infty}(\Omega)$ can be defined with the usual modification. Sometimes, we shall also use the 3D Lebesgue measure $r d r d z$, and the corresponding Lebesgue spaces are then denoted by $L^{p}\left(\mathbb{R}^{3}\right)$ or $L^{p}$ with norm

$$
\|g\|_{L^{p}} \stackrel{\text { def }}{=}\left(\int_{\Omega}|f(r, z)|^{p} r d r d z\right)^{\frac{1}{p}}, 1 \leq p<\infty .
$$

Our main results state as follows.

Theorem 1.1. For any initial data $\omega_{0}^{\theta} \in L^{1}(\Omega)$ and $u_{0}^{\theta} \in L^{2}(\Omega)$ satisfying $r^{-\frac{3}{10}} u_{0}^{\theta} \in L^{\frac{20}{13}}(\Omega)$, there exists some $T\left(\omega_{0}^{\theta}, u_{0}^{\theta}\right)$ such that the equations (1.4) have a unique mild solution

$$
\begin{gathered}
\left.\left.\left.\left.\omega^{\theta} \in C\left([0, T] ; L^{1}(\Omega)\right) \bigcap C(] 0, T\right] ; L^{\infty}(\Omega)\right), u^{\theta} \in C\left([0, T] ; L^{2}(\Omega)\right) \bigcap C(] 0, T\right] ; L^{\infty}(\Omega)\right) \\
\text { with } \left.\left.\quad r^{-\frac{3}{10}} u^{\theta} \in C\left([0, T] ; L^{\frac{20}{13}}(\Omega)\right) \bigcap C(] 0, T\right] ; L^{\infty}(\Omega)\right) .
\end{gathered}
$$

Furthermore, the solution $\left(\omega^{\theta}, u^{\theta}\right)$ verifies

- for any $p \in[1, \infty], q \in[2, \infty]$ and $\kappa \in[20 / 13, \infty]$, there holds

$$
\begin{aligned}
& L_{p}(T) \stackrel{\text { def }}{=} \sup _{0 \leq t \leq T} t^{1-\frac{1}{p}}\left\|\omega^{\theta}(t)\right\|_{L^{p}(\Omega)}<\infty, \quad M_{q}(T) \stackrel{\text { def }}{=} \sup _{0 \leq t \leq T} t^{\frac{1}{2}-\frac{1}{q}}\left\|u^{\theta}(t)\right\|_{L^{q}(\Omega)}<\infty, \\
& N_{\kappa}(T) \stackrel{\text { def }}{=} \sup _{0 \leq t \leq T} t^{\frac{13}{20}-\frac{1}{\kappa}}\left\|r^{-\frac{3}{10}} u^{\theta}(t)\right\|_{L^{\kappa}(\Omega)}<\infty .
\end{aligned}
$$

Moreover, when $p \in] 1, \infty], q \in] 2, \infty]$ and $\kappa \in] 20 / 13, \infty]$, we have

$$
\lim _{t \rightarrow 0}\left(L_{p}(t)+M_{q}(t)+N_{\kappa}(t)\right)=0 ;
$$

- if

$$
\left\|\omega_{0}^{\theta}\right\|_{L^{1}(\Omega)}+\left\|u_{0}^{\theta}\right\|_{L^{2}(\Omega)}+\left\|r^{-\frac{3}{10}} u_{0}^{\theta}\right\|_{L^{\frac{20}{13}(\Omega)}} \leq c
$$

for some sufficiently small constant $c$, then $T=\infty$. And if $\left\|u_{0}^{\theta}\right\|_{L^{2}(\Omega)}+\left\|r^{-\frac{3}{10}} u_{0}^{\theta}\right\|_{L^{\frac{20}{13}}(\Omega)}$ is small enough, then the lifespan $T^{\star}$ of the solution depends only on $\omega_{0}^{\theta}$. 
Remark 1.1. - Let us remark that the norms $\left\|\omega_{0}^{\theta}\right\|_{L^{1}(\Omega)},\left\|u_{0}^{\theta}\right\|_{L^{2}(\Omega)}$ and $\left\|r^{-\frac{3}{10}} u_{0}^{\theta}\right\|_{L^{\frac{20}{13}}(\Omega)}$ are scaling invariant under the scaling transformation (1.2). Moreover, the method used here might be used to study axi-symmetric vortex ring for 3-D Navier-Stokes system with swirl (see the corresponding result of [4] for the case without swirl).

- The reason for requiring $r^{-\frac{3}{10}} u_{0}^{\theta} \in L^{\frac{20}{13}}(\Omega)$ is to handle the term $\frac{\partial_{z}\left|u^{\theta}(s)\right|^{2}}{r}$ in $\omega^{\theta}$ equation of (3.1), so that the exponent $\frac{3}{2}-\frac{1}{p}+\frac{1}{5}$ appearing in (3.7) is less than 1.

Theorem 1.2. Let $\omega_{0}^{\theta}$ and $u_{0}^{\theta}$ satisfy $\eta_{0} \stackrel{\text { def }}{=} \frac{\omega_{0}^{\theta}}{r} \in L^{1}, U_{0} \stackrel{\text { def }}{=} \frac{u_{0}^{\theta}}{r} \in L^{\frac{3}{2}}, r u_{0}^{\theta} \in L^{A} \cap L^{\infty}$ for some finite $A$. We assume that $\left\|r u_{0}^{\theta}\right\|_{L^{\infty}(\Omega)}$ is sufficiently small, then the system (1.4) has a unique global solution which satisfies

$$
\eta \stackrel{\text { def }}{=} \frac{\omega^{\theta}}{r} \in C\left(\left[0 , + \infty [ ; L ^ { 1 } ) \quad \text { and } \quad U \stackrel { \text { def } } { = } \frac { u ^ { \theta } } { r } \in C \left(\left[0,+\infty\left[; L^{\frac{3}{2}}\right) .\right.\right.\right.\right.
$$

Remark 1.2. - The main difficulty in the proof of the above theorem is when $\omega_{0}^{\theta} \in$ $L^{p}(\Omega)$ for $p=1$, the dissipative term, $\frac{4(p-1)}{p^{2}}\left\|\nabla|\eta|^{\frac{p}{2}}\right\|_{L^{2}}^{2}$, in (4.7) disappears. That is the reason why we divide the proof of Theorem 1.2 in the following two steps: we first get, by applying Theorem 1.1, that the system (1.4) has a unique local solution with $\eta\left(t_{0}\right) \in L^{p_{0}}\left(\mathbb{R}^{3}\right)$ for some $t_{0}>0$ and $p_{0}>1$; then in the second step, starting with initial data at $t_{0}$, we prove the global well-posedness of the system (1.4).

- One may see (5.6), (5.17), (5.19) and (5.24) for the exact smallness condition for $\left\|r u_{0}^{\theta}\right\|_{L^{\infty}}$. And the exact global estimate of $\|\eta(t)\|_{L^{1}}$ and $\|U(t)\|_{L^{\frac{3}{2}}}$ is given in (5.25).

- It follows from Lemma 2.1 below and Hölder's inequality

$$
\left.\left\|r^{\kappa} u^{\theta}\right\|_{L^{\frac{3}{1-\kappa}}} \leq\|U\|_{L^{\frac{3}{2}}}^{\frac{1-\kappa}{2}}\left\|r u^{\theta}\right\|_{L^{\infty}}^{\frac{1+\kappa}{2}}, \quad \forall \kappa \in\right]-1,1[,
$$

that the solutions constructed in Theorem 1.2 in fact satisfy

$$
r^{\kappa} u^{\theta} \in C\left(\left[0,+\infty\left[; L^{\frac{3}{1-\kappa}}\right), \quad \forall \kappa \in[-1,1] .\right.\right.
$$

\section{PReliminaries}

\subsection{Some elementary results.}

Lemma 2.1 (Proposition 1 of [2]). Let $\left(u^{r}, u^{\theta}, u^{z}\right)$ be a smooth enough solution of (1.3) on $[0, T]$. Then for any $p \in[2, \infty]$, we have

$$
\left\|r u^{\theta}(t)\right\|_{L^{p}} \leqslant\left\|r u_{0}^{\theta}\right\|_{L^{p}} \quad \forall t \in[0, T] .
$$

Lemma 2.2 (See Lemma 5.5 from [1] for instance). Let $E$ be a Banach space, $\mathfrak{B}(\cdot, \cdot)$ a continuous bilinear map from $E \times E$ to $E$, and $\alpha$ a positive real number such that

$$
\alpha<\frac{1}{4\|\mathfrak{B}\|} \quad \text { with } \quad\|\mathfrak{B}\| \stackrel{\text { def }}{=} \sup _{\|f\|,\|g\| \leq 1}\|\mathfrak{B}(f, g)\| .
$$

Then for any $a$ in the ball $B(0, \alpha)$ in $E$, there exists a unique $x$ in $B(0,2 \alpha)$ such that

$$
x=a+\mathfrak{B}(x, x) .
$$

Let us recall also some facts from Section 2 of [5]. We first recall the axisymmetric BiotSavart law which determines $\widetilde{u}=\left(u^{r}, u^{z}\right)$ in terms of $\omega^{\theta}$, namely

$$
u^{r}(r, z)=\int_{\Omega} G_{r}(r, z, \bar{r}, \bar{z}) \omega^{\theta}(\bar{r}, \bar{z}) d \bar{r} d \bar{z}, \quad u^{z}(r, z)=\int_{\Omega} G_{z}(r, z, \bar{r}, \bar{z}) \omega^{\theta}(\bar{r}, \bar{z}) d \bar{r} d \bar{z}
$$


where

$$
\begin{aligned}
G_{r}(r, z, \bar{r}, \bar{z}) & =-\frac{1}{\pi} \frac{z-\bar{z}}{r^{3 / 2} \bar{r}^{1 / 2}} F^{\prime}\left(\xi^{2}\right), \quad \xi^{2}=\frac{(r-\bar{r})^{2}+(z-\bar{z})^{2}}{r \bar{r}}, \\
G_{z}(r, z, \bar{r}, \bar{z}) & =\frac{1}{\pi} \frac{r-\bar{r}}{r^{3 / 2} \bar{r}^{1 / 2}} F^{\prime}\left(\xi^{2}\right)+\frac{1}{4 \pi} \frac{\bar{r}^{1 / 2}}{r^{3 / 2}}\left(F\left(\xi^{2}\right)-2 \xi^{2} F^{\prime}\left(\xi^{2}\right)\right) \quad \text { with } \\
F(s) & =\int_{0}^{\frac{\pi}{2}} \frac{\cos (2 \phi) d \phi}{\left(\sin ^{2} \phi+s / 4\right)^{1 / 2}}, \quad s>0 .
\end{aligned}
$$

It follows from the Remark 2.2 of [5] that

Lemma 2.3. $s^{\alpha} F(s)$ and $s^{\beta} F^{\prime}(s)$ are bounded on $] 0, \infty[$ for $\left.\alpha \in] 0,3 / 2\right]$ and $\beta \in[1,5 / 2]$.

Lemma 2.4 (Proposition 2.3 of [5]). Let us denote $\widetilde{u} \stackrel{\text { def }}{=}\left(u^{r}, u^{z}\right)$. Then one has

i) Assume that $1<p<2<q<\infty$ and $\frac{1}{q}=\frac{1}{p}-\frac{1}{2}$. If $\omega^{\theta} \in L^{p}(\Omega)$, then $\widetilde{u} \in L^{q}(\Omega)$ and

$$
\|\widetilde{u}\|_{L^{q}(\Omega)} \leqslant C\left\|\omega^{\theta}\right\|_{L^{p}(\Omega)} .
$$

ii) If $1 \leqslant p<2<q \leqslant \infty$ and $\omega^{\theta} \in L^{p}(\Omega) \bigcap L^{q}(\Omega)$, then $\widetilde{u} \in L^{\infty}(\Omega)$ and

$$
\left.\|\widetilde{u}\|_{L^{\infty}(\Omega)} \leqslant C\left\|\omega^{\theta}\right\|_{L^{p}(\Omega)}^{\sigma}\left\|\omega^{\theta}\right\|_{L^{q}(\Omega)}^{1-\sigma}, \quad \text { where } \quad \sigma=\frac{p(q-2)}{2(q-p)} \in\right] 0,1[.
$$

Next we investigate the solution operator $S(t)$ to the linearized system of (1.4), namely $\omega^{\theta}(t)=S(t) \omega_{0}$ verifies

$$
\left\{\begin{array}{l}
\partial_{t} \omega^{\theta}-\left(\partial_{r}^{2}+\partial_{z}^{2}+\frac{1}{r} \partial_{r}-\frac{1}{r^{2}}\right) \omega^{\theta}=0, \quad(t, r, z) \in \mathbb{R}^{+} \times \Omega \\
\left.\omega^{\theta}\right|_{r=0}=0, \\
\left.\omega^{\theta}\right|_{t=0}=\omega_{0}^{\theta} .
\end{array}\right.
$$

Lemma 2.5 (Lemma 3.1, 3.2 of [5]). For any $t>0$, one has

$$
\left(S(t) \omega_{0}\right)(r, z)=\frac{1}{4 \pi t} \int_{\Omega} \frac{\bar{r}^{1 / 2}}{r^{1 / 2}} H\left(\frac{t}{r \bar{r}}\right) \exp \left(-\frac{(r-\bar{r})^{2}+(z-\bar{z})^{2}}{4 t}\right) \omega_{0}(\bar{r}, \bar{z}) d \bar{r} d \bar{z},
$$

where the function $H:] 0,+\infty[\rightarrow \mathbb{R}$ is defined by

$$
H(t)=\frac{1}{\sqrt{\pi t}} \int_{-\pi / 2}^{\pi / 2} e^{-\frac{\sin ^{2} \phi}{t}} \cos (2 \phi) d \phi, \quad t>0,
$$

which is smooth on $] 0, \infty[$ and has the asymptotic expansions:

i) $H(t)=\frac{\pi^{1 / 2}}{4 t^{3 / 2}}+\mathcal{O}\left(\frac{1}{t^{5 / 2}}\right), H^{\prime}(t)=-\frac{3 \pi^{1 / 2}}{8 t^{5 / 2}}+\mathcal{O}\left(\frac{1}{t^{7 / 2}}\right)$, as $t \rightarrow \infty$;

ii) $H(t)=1-\frac{3 t}{4}+\mathcal{O}\left(t^{2}\right), H^{\prime}(t)=-\frac{3}{4}+\mathcal{O}(t)$, as $t \rightarrow 0$.

Corollary 2.1. $t^{\alpha} H(t)$ and $t^{\beta} H^{\prime}(t)$ are bounded on $] 0, \infty\left[\right.$ provided $0 \leq \alpha \leq \frac{3}{2}, 0 \leq \beta \leq \frac{5}{2}$.

2.2. The estimate of $\frac{u^{r}}{r}$ in terms of $\frac{\omega^{\theta}}{r}$. In this subsection, we shall exploit the basic facts recalled in Subsection 2.1 to derive the estimate of $\frac{u^{r}}{r}$ in terms of $\frac{\omega^{\theta}}{r}$, which will be used in Section 4 below. The main result states as follows:

Proposition 2.1. Let $p \in] 1,3[$ and $\left.q \in] \frac{3 p}{3-p}, \infty\right]$. We assume that $\eta \stackrel{\text { def }}{=} \frac{\omega^{\theta}}{r} \in L^{p}\left(\mathbb{R}^{3}\right) \cap$ $L^{3 p}\left(\mathbb{R}^{3}\right)$. Then we have

$$
\left\|\frac{u^{r}}{r}\right\|_{L^{q}} \lesssim\|\eta\|_{L^{p}}^{\lambda}\|\eta\|_{L^{3 p}}^{1-\lambda} \quad \text { with } \quad \lambda=\frac{p-1}{2}+\frac{3 p}{2 q} .
$$


Proof. By virtue of (2.2) and (2.3), we write

$$
r^{\frac{1}{q}} \frac{u^{r}(r, z)}{r}=-\int_{\Omega} \frac{z-\bar{z}}{\pi} \frac{\bar{r}^{\frac{1}{2}}}{r^{\frac{5}{2}-\frac{1}{q}}} F^{\prime}\left(\xi^{2}\right) \eta(\bar{r}, \bar{z}) d \bar{r} d \bar{z} \quad \forall(r, z) \in \Omega .
$$

We decompose the integral domain $\Omega=I_{1} \bigcup I_{2}$ with

$$
I_{1} \stackrel{\text { def }}{=}\{(\bar{r}, \bar{z}) \in \Omega \mid \bar{r} \leq 2 r,\} \quad \text { and } \quad I_{2} \stackrel{\text { def }}{=} \Omega \backslash I_{1} .
$$

We first consider the case when $q<\infty$. Let $s$ be determined by $\frac{1}{s}=\frac{1}{q}+\frac{1}{3}$. Then due to $q \in] \frac{3 p}{3-p}, \infty\left[\right.$, we have $s>p$. Moreover, it follows from Lemma 2.3 that $\left|F^{\prime}(s)\right| \lesssim\left(\frac{1}{s}\right)^{\frac{7}{6}}=$ $\left(\frac{1}{s}\right)^{1+\frac{1}{2}\left(\frac{1}{s}-\frac{1}{q}\right)}$. Note that that $\frac{\bar{r}}{r} \leqslant 2$ in $I_{1}$ and $\frac{3}{2}-\frac{1}{2}\left(\frac{1}{s}+\frac{1}{q}\right)>0$, we thus obtain

$$
\begin{aligned}
& \left|\int_{I_{1}} \frac{z-\bar{z}}{\pi} \frac{\bar{r}^{\frac{1}{2}}}{r^{\frac{5}{2}-\frac{1}{q}}} F^{\prime}\left(\xi^{2}\right) \eta(\bar{r}, \bar{z}) d \bar{r} d \bar{z}\right| \\
& \lesssim \int_{I_{1}} \frac{|z-\bar{z}| \cdot \bar{r}^{\frac{1}{2}-\frac{1}{s}}}{r^{\frac{5}{2}-\frac{1}{q}}}\left(\frac{r \bar{r}}{(r-\bar{r})^{2}+(z-\bar{z})^{2}}\right)^{\frac{7}{6}} \cdot \bar{r}^{\frac{1}{s}}|\eta(\bar{r}, \bar{z})| d \bar{r} d \bar{z} \\
& \lesssim \int_{I_{1}}\left(\frac{\bar{r}}{r}\right)^{\frac{3}{2}-\frac{1}{2}\left(\frac{1}{s}+\frac{1}{q}\right)}\left(\frac{1}{|(\bar{r}, \bar{z})-(r, z)|}\right)^{\frac{4}{3}} \cdot \bar{r}^{\frac{1}{s}}|\eta(\bar{r}, \bar{z})| d \bar{r} d \bar{z} \\
& \lesssim \int_{\Omega}\left(\frac{1}{|(\bar{r}, \bar{z})-(r, z)|}\right)^{\frac{4}{3}} \cdot \bar{r}^{\frac{1}{s}}|\eta(\bar{r}, \bar{z})| d \bar{r} d \bar{z},
\end{aligned}
$$

from which, and Hardy-Littlewood-Sobolev inequality, we infer

$$
\begin{aligned}
& \left\|\int_{I_{1}} \frac{z-\bar{z}}{\pi} \frac{\bar{r}^{\frac{1}{2}}}{r^{\frac{5}{2}-\frac{1}{q}}} F^{\prime}\left(\xi^{2}\right) \eta(\bar{r}, \bar{z}) d \bar{r} d \bar{z}\right\|_{L^{q}(\Omega ; d r d z)} \\
& \lesssim\left\||(r, z)|^{-\frac{4}{3}}\right\|_{L^{\frac{3}{2}, \infty}(\Omega)}\left\|r^{\frac{1}{s}} \eta\right\|_{L^{s}(\Omega)} \\
& \sim\|\eta\|_{L^{s}} \lesssim\|\eta\|_{L^{p}}^{\frac{p-1}{2}+\frac{3 p}{2 q}}\|\eta\|_{L^{3 p}}^{1-\frac{p-1}{2}-\frac{3 p}{2 q}} .
\end{aligned}
$$

Note that in the region $I_{2}$, there holds $\bar{r} \leq 2|\bar{r}-r|$. Thus by using Lemma 2.3, we get

$$
\begin{aligned}
\left|\int_{I_{2}} \frac{z-\bar{z}}{\pi} \frac{\bar{r}^{\frac{1}{2}}}{r^{\frac{5}{2}-\frac{1}{q}}} F^{\prime}\left(\xi^{2}\right) \eta(\bar{r}, \bar{z}) d \bar{r} d \bar{z}\right| & \lesssim \int_{I_{2}} \frac{|z-\bar{z}| \cdot \bar{r}^{\frac{1}{2}}}{r^{\frac{5}{2}-\frac{1}{q}}}\left(\frac{r \bar{r}}{(r-\bar{r})^{2}+(z-\bar{z})^{2}}\right)^{\frac{5}{2}-\frac{1}{q}} \cdot|\eta(\bar{r}, \bar{z})| d \bar{r} d \bar{z} \\
& \lesssim \int_{I_{2}}\left(\frac{1}{|(\bar{r}, \bar{z})-(r, z)|}\right)^{1-\frac{1}{q}}|\eta(\bar{r}, \bar{z})| d \bar{r} d \bar{z} .
\end{aligned}
$$

To proceed further, for any given $R>0$, we split $I_{2}=I_{21} \cup I_{22}$ with $I_{21}=I_{2} \cap\{(\bar{r}, \bar{z}) \in \Omega||(\bar{r}, \bar{z})-(r, z) \mid \geq R\}, \quad I_{22}=I_{2} \cap\{(\bar{r}, \bar{z}) \in \Omega||(\bar{r}, \bar{z})-(r, z) \mid<R\}$.

Then we get, by applying Young's inequality, that

$$
\begin{aligned}
& \left\|\int_{I_{21}} \frac{z-\bar{z}}{\pi} \frac{\bar{r}^{\frac{1}{2}}}{r^{\frac{5}{2}-\frac{1}{q}}} F^{\prime}\left(\xi^{2}\right) \eta(\bar{r}, \bar{z}) d \bar{r} d \bar{z}\right\|_{L^{q}(\Omega)} \\
& \leq\left\|\int_{I_{21}}\left(\frac{1}{|(\bar{r}, \bar{z})-(r, z)|}\right)^{1+\frac{1}{p}-\frac{1}{q}} \cdot \bar{r}^{\frac{1}{p}}|\eta(\bar{r}, \bar{z})| d \bar{r} d \bar{z}\right\|_{L^{q}(\Omega)} \\
& \lesssim\left\|r^{\frac{1}{p}} \eta\right\|_{L^{p}(\Omega)}\left(\int_{R}^{\infty} \rho^{\frac{2 / q-2 / p}{1-1 / p+1 / q}} \rho d \rho\right)^{1-\frac{1}{p}+\frac{1}{q}} \sim R^{1-\frac{3}{p}+\frac{3}{q}}\|\eta\|_{L^{p}}
\end{aligned}
$$


For the integral on $I_{22}$, in the case $q>3 p$, by applying Young's inequality, we get

$$
\begin{aligned}
& \left\|\int_{I_{22}} \frac{z-\bar{z}}{\pi} \frac{\bar{r}^{\frac{1}{2}}}{r^{\frac{5}{2}-\frac{1}{q}}} F^{\prime}\left(\xi^{2}\right) \eta(\bar{r}, \bar{z}) d \bar{r} d \bar{z}\right\|_{L^{q}(\Omega)} \\
& \leq\left\|\int_{I_{22}}\left(\frac{1}{|(\bar{r}, \bar{z})-(r, z)|}\right)^{1+\frac{1}{3 p}-\frac{1}{q}} \cdot \bar{r}^{\frac{1}{3 p}}|\eta(\bar{r}, \bar{z})| d \bar{r} d \bar{z}\right\|_{L^{q}(\Omega)} \\
& \lesssim\left\|r^{\frac{1}{3 p}} \eta\right\|_{L^{3 p}(\Omega)}\left(\int_{0}^{R} \rho^{\frac{2 / q-2 / 3 p}{1-1 / 3 p+1 / q}} d \rho\right)^{1-\frac{1}{3 p}+\frac{1}{q}} \sim R^{1-\frac{1}{p}+\frac{3}{q}}\|\eta\|_{L^{3 p}} .
\end{aligned}
$$

While in the case $\frac{3 p}{3-p}<q \leq 3 p$, another use of Young's inequality gives

$$
\begin{aligned}
& \left\|\int_{I_{22}} \frac{z-\bar{z}}{\pi} \frac{\bar{r}^{\frac{1}{2}}}{r^{\frac{5}{2}-\frac{1}{q}}} F^{\prime}\left(\xi^{2}\right) \eta(\bar{r}, \bar{z}) d \bar{r} d \bar{z}\right\|_{L^{q}(\Omega)} \\
& \leq\left\|\int_{I_{22}}\left(\frac{1}{|(\bar{r}, \bar{z})-(r, z)|}\right)^{1+\frac{3-p}{3 p}-\frac{1}{q}} \cdot \bar{r}^{\frac{3-p}{3 p}}|\eta(\bar{r}, \bar{z})| d \bar{r} d \bar{z}\right\|_{L^{q}(\Omega)} \\
& \lesssim\left\|r^{\frac{3-p}{3 p}} \eta\right\|_{L^{\frac{3 p}{3-p}}(\Omega)}\left(\int_{0}^{R} \rho^{\frac{2 / q-2(3-p) / 3 p}{1-(3-p) / 3 p+1 / q}} d \rho\right)^{1-\frac{3-p}{3 p}+\frac{1}{q}} \\
& \sim R^{2-\frac{3}{p}+\frac{3}{q}}\|\eta\|_{L^{\frac{3 p}{3-p}}} \lesssim R^{1-\frac{3}{p}+\frac{3}{q}}\|\eta\|_{L^{p}}+R^{1-\frac{1}{p}+\frac{3}{q}}\|\eta\|_{L^{3 p}} .
\end{aligned}
$$

As a result, it comes out

$$
\left\|\int_{I_{2}} \frac{z-\bar{z}}{\pi} \frac{\bar{r}^{\frac{1}{2}}}{r^{\frac{5}{2}-\frac{1}{q}}} F^{\prime}\left(\xi^{2}\right) \eta(\bar{r}, \bar{z}) d \bar{r} d \bar{z}\right\|_{L^{q}(\Omega)} \lesssim R^{1-\frac{3}{p}+\frac{3}{q}}\|\eta\|_{L^{p}}+R^{1-\frac{1}{p}+\frac{3}{q}}\|\eta\|_{L^{3 p}} .
$$

Taking $R=\left(\frac{\|\eta\|_{L} p}{\|\eta\|_{L} 3 p}\right)^{\frac{p}{2}}$ in the above inequality gives rise to

$$
\left\|\int_{I_{2}} \frac{z-\bar{z}}{\pi} \frac{\bar{r}^{\frac{1}{2}}}{r^{\frac{5}{2}-\frac{1}{q}}} F^{\prime}\left(\xi^{2}\right) \eta(\bar{r}, \bar{z}) d \bar{r} d \bar{z}\right\|_{L^{q}(\Omega)} \lesssim\|\eta\|_{L^{p}}^{\frac{p-1}{2}+\frac{3 p}{2 q}}\|\eta\|_{L^{3 p}}^{1-\frac{p-1}{2}-\frac{3 p}{2 q}} .
$$

Due to $\left\|r^{\frac{1}{q}} \frac{u^{r}}{r}\right\|_{L^{q}(\Omega)}=\left\|\frac{u^{r}}{r}\right\|_{L^{q}},(2.12)$ together with (2.13) ensures (2.9) for any $\left.q \in\right] \frac{3 p}{3-p}, \infty[$.

The end-point case when $q=\infty$ follows exactly along the same line. This completes the proof of Proposition 2.1.

2.3. The estimates of the solution operator $S(t)$. The goal of this subsection is to present the estimates of the solution operator $S(t)$, which will be used in Section 3 .

Proposition 2.2. Let $S(t)$ the solution operator given by (2.7). Then this family $(S(t))_{t>0}$ are strongly continuous semigroups of bounded linear operators in $L^{m}(\Omega)$ for any $m \in[1, \infty[$. Moreover, for $1 \leq p \leq q \leq \infty$, there holds

(1) For any $\alpha, \beta$ satisfying $\alpha+\beta \leq 0, a \geq-1$ and $\beta \geq-1$, and any $f=\left(f^{r}, f^{z}\right) \in L^{p}(\Omega)^{2}$, there holds

$$
\left\|r^{\alpha} S(t) \operatorname{div}_{*}\left(r^{\beta} f\right)\right\|_{L^{q}(\Omega)} \leq \frac{C}{t^{\frac{1}{2}-\frac{\alpha+\beta}{2}+\frac{1}{p}-\frac{1}{q}}}\|f\|_{L^{p}(\Omega)} .
$$

In particular, taking $\alpha=\beta=0$, we have

$$
\left\|S(t) \operatorname{div}_{*} f\right\|_{L^{q}(\Omega)} \leq \frac{C}{t^{\frac{1}{2}+\frac{1}{p}-\frac{1}{q}}}\|f\|_{L^{p}(\Omega)} .
$$


(2) For any $\alpha, \beta$ satisfying $\alpha+\beta \leq 1, \alpha \geq-1$ and $\beta \geq-1$, and any $g \in L^{p}(\Omega)$, there holds

$$
\left\|r^{\alpha} S(t)\left(r^{\beta-1} g\right)\right\|_{L^{q}(\Omega)} \leq \frac{C}{t^{\frac{1}{2}-\frac{\alpha+\beta}{2}+\frac{1}{p}-\frac{1}{q}}}\|g\|_{L^{p}(\Omega)},
$$

In particular, taking $\alpha=0, \beta=1$, and $\alpha=\beta=0$, we have

$$
\|S(t) g\|_{L^{q}(\Omega)} \leq \frac{C}{t^{\frac{1}{p}-\frac{1}{q}}}\|g\|_{L^{p}(\Omega)}, \quad\left\|S(t)\left(\frac{g}{r}\right)\right\|_{L^{q}(\Omega)} \leq \frac{C}{t^{\frac{1}{2}+\frac{1}{p}-\frac{1}{q}}}\|g\|_{L^{p}(\Omega)}
$$

(3) For any $\delta \in\left[-1, \frac{1}{2}\right], m \in\left[1, \infty\left[\right.\right.$, and any $g$ satisfying $r^{\delta} g \in L^{m}(\Omega)$, we have

$$
\left\|r^{\delta} S(t) g-r^{\delta} g\right\|_{L^{m}(\Omega)} \rightarrow 0, \quad \text { as } t \rightarrow 0 .
$$

Proof. The boundedness of the semigroup $(S(t))_{t \geq 0}$ is shown in (2.17). Then in order to prove $(S(t))_{t>0}$ is strongly continuous in $L^{m}(\Omega)$ for any $m \in[1, \infty[$, we only need to verify the continuity at the origin, which is a direct consequence of (2.18) (with $\delta=0$ ). Hence it remains to prove the estimates $(2.14-2.17)$, which we handle term by term below.

(1) By integration by parts, we write

$$
\begin{aligned}
& r^{\alpha}\left(S(t) \operatorname{div}_{*}\left(r^{\beta} f\right)\right)(r, z) \\
& \quad=\frac{1}{4 \pi t} \int_{\Omega} \frac{\bar{r}^{\frac{1}{2}+\beta}}{r^{\frac{1}{2}-\alpha}} \exp \left(-\frac{(r-\bar{r})^{2}+(z-\bar{z})^{2}}{4 t}\right) \cdot\left(A_{r} f^{r}+A_{z} f^{z}\right)(\bar{r}, \bar{z}) d \bar{r} d \bar{z},
\end{aligned}
$$

where

$$
A_{r}(\bar{r}, \bar{z})=\frac{t}{r \bar{r}^{2}} H^{\prime}\left(\frac{t}{r \bar{r}}\right)-\left(\frac{1}{2 \bar{r}}+\frac{r-\bar{r}}{2 t}\right) H\left(\frac{t}{r \bar{r}}\right), \quad A_{z}(\bar{r}, \bar{z})=-\frac{z-\bar{z}}{2 t} H\left(\frac{t}{r \bar{r}}\right) .
$$

- Let us first handle the term $\left|A_{r}+\frac{r-\bar{r}}{2 t} H\left(\frac{t}{r \bar{r}}\right)\right|$.

If $(\alpha, \beta) \in \Omega_{1} \stackrel{\text { def }}{=}\left\{(\alpha, \beta) \mid 0 \leq \frac{1}{2}-\frac{\alpha+\beta}{2} \leq \frac{3}{2}, 0 \leq \frac{1}{2}-\beta \leq \frac{3}{2}, \beta \leq \alpha\right\}$, we can divide the integral area into $\left\{\bar{r} \geq \frac{r}{2}\right\}$ and $\left\{\bar{r}<\frac{r}{2}\right\}$. When $\bar{r} \geq \frac{r}{2}$, we can deduce from Corollary 2.1 that

$$
\begin{aligned}
& \frac{\bar{r}^{\frac{1}{2}+\beta}}{r^{\frac{1}{2}-\alpha}} \exp \left(-\frac{(r-\bar{r})^{2}+(z-\bar{z})^{2}}{4 t}\right)\left|A_{r}+\frac{r-\bar{r}}{2 t} H\left(\frac{t}{r \bar{r}}\right)\right| \\
& \lesssim\left(\frac{t}{r^{\frac{3}{2}-\alpha} \bar{r}^{\frac{3}{2}-\beta}} H^{\prime}\left(\frac{t}{r \bar{r}}\right)+\frac{1}{r^{\frac{1}{2}-\alpha} \bar{r}^{\frac{1}{2}-\beta}} H\left(\frac{t}{r \bar{r}}\right)\right) \cdot \exp \left(-\frac{(r-\bar{r})^{2}+(z-\bar{z})^{2}}{4 t}\right) \\
& \lesssim\left(\frac{t}{r^{\frac{3}{2}-\alpha} \bar{r}^{\frac{3}{2}-\beta}}\left|\frac{r \bar{r}}{t}\right|^{\frac{3}{2}-\frac{\alpha+\beta}{2}}+\frac{1}{r^{\frac{1}{2}-\alpha} \bar{r}^{\frac{1}{2}-\beta}}\left|\frac{r \bar{r}}{t}\right|^{\frac{1}{2}-\frac{\alpha+\beta}{2}}\right) \cdot \exp \left(-\frac{(r-\bar{r})^{2}+(z-\bar{z})^{2}}{4 t}\right) \\
& \lesssim \frac{1}{t^{\frac{1}{2}-\frac{\alpha+\beta}{2}}} \cdot \exp \left(-\frac{(r-\bar{r})^{2}+(z-\bar{z})^{2}}{5 t}\right) .
\end{aligned}
$$


And when $\bar{r}<\frac{r}{2}$, there then holds $r<2|\bar{r}-r|$, another use of Corollary 2.1 gives

$$
\begin{aligned}
& \frac{\bar{r}^{\frac{1}{2}+\beta}}{r^{\frac{1}{2}-\alpha}} \exp \left(-\frac{(r-\bar{r})^{2}+(z-\bar{z})^{2}}{4 t}\right)\left|A_{r}+\frac{r-\bar{r}}{2 t} H\left(\frac{t}{r \bar{r}}\right)\right| \\
& \lesssim\left(\frac{t}{r^{\frac{3}{2}-\alpha} \bar{r}^{\frac{3}{2}-\beta}}\left|\frac{r \bar{r}}{t}\right|^{\frac{3}{2}-\beta}+\frac{1}{r^{\frac{1}{2}-\alpha} \bar{r}^{\frac{1}{2}-\beta}}\left|\frac{r \bar{r}}{t}\right|^{\frac{1}{2}-\beta}\right) \exp \left(-\frac{(r-\bar{r})^{2}+(z-\bar{z})^{2}}{4 t}\right) \\
& \lesssim \frac{r^{\alpha-\beta}}{t^{\frac{1}{2}-\beta}} \cdot\left(\frac{5 t}{(r-\bar{r})^{2}+(z-\bar{z})^{2}}\right)^{\frac{\alpha-\beta}{2}} \cdot \exp \left(-\frac{(r-\bar{r})^{2}+(z-\bar{z})^{2}}{5 t}\right) \\
& \lesssim \frac{1}{t^{\frac{1}{2}-\frac{\alpha+\beta}{2}}} \cdot \exp \left(-\frac{(r-\bar{r})^{2}+(z-\bar{z})^{2}}{5 t}\right) \text {. }
\end{aligned}
$$

If $(\alpha, \beta) \in \Omega_{2} \stackrel{\text { def }}{=}\left\{(\alpha, \beta) \mid 0 \leq \frac{1}{2}-\frac{\alpha+\beta}{2} \leq \frac{3}{2}, 0 \leq \frac{1}{2}-\alpha \leq \frac{3}{2}, \alpha \leq \beta\right\}$, we divide the integral area in a different way as $\{\bar{r} \leq 2 r\}$ and $\{\bar{r}>2 r\}$. Similar to the previous estimates, when $\bar{r} \leq 2 r$, we have

$$
\begin{aligned}
& \frac{\bar{r}^{\frac{1}{2}+\beta}}{r^{\frac{1}{2}-\alpha}} \exp \left(-\frac{(r-\bar{r})^{2}+(z-\bar{z})^{2}}{4 t}\right)\left|A_{r}+\frac{r-\bar{r}}{2 t} H\left(\frac{t}{r \bar{r}}\right)\right| \\
& \lesssim\left(\frac{t}{r^{\frac{3}{2}-\alpha} \bar{r}^{\frac{3}{2}-\beta}}\left|\frac{r \bar{r}}{t}\right|^{\frac{3}{2}-\frac{\alpha+\beta}{2}}+\frac{1}{r^{\frac{1}{2}-\alpha} \bar{r}^{\frac{1}{2}-\beta}}\left|\frac{r \bar{r}}{t}\right|^{\frac{1}{2}-\frac{\alpha+\beta}{2}}\right) \cdot \exp \left(-\frac{(r-\bar{r})^{2}+(z-\bar{z})^{2}}{4 t}\right) \\
& \lesssim \frac{1}{t^{\frac{1}{2}-\frac{\alpha+\beta}{2}}} \cdot \exp \left(-\frac{(r-\bar{r})^{2}+(z-\bar{z})^{2}}{5 t}\right) .
\end{aligned}
$$

And when $\bar{r}>2 r$, there then holds $\bar{r}<2|\bar{r}-r|$, thus we deduce

$$
\begin{aligned}
& \frac{\bar{r}^{\frac{1}{2}+\beta}}{r^{\frac{1}{2}-\alpha}} \exp \left(-\frac{(r-\bar{r})^{2}+(z-\bar{z})^{2}}{4 t}\right)\left|A_{r}+\frac{r-\bar{r}}{2 t} H\left(\frac{t}{r \bar{r}}\right)\right| \\
& \lesssim\left(\frac{t}{r^{\frac{3}{2}-\alpha} \bar{r}^{\frac{3}{2}-\beta}}\left|\frac{r \bar{r}}{t}\right|^{\frac{3}{2}-\alpha}+\frac{1}{r^{\frac{1}{2}-\alpha} \bar{r}^{\frac{1}{2}-\beta}}\left|\frac{r \bar{r}}{t}\right|^{\frac{1}{2}-\alpha}\right) \exp \left(-\frac{(r-\bar{r})^{2}+(z-\bar{z})^{2}}{4 t}\right) \\
& \lesssim \frac{\bar{r}^{\beta-\alpha}}{t^{\frac{1}{2}-\alpha}} \cdot\left(\frac{5 t}{(r-\bar{r})^{2}+(z-\bar{z})^{2}}\right)^{\frac{\beta-\alpha}{2}} \cdot \exp \left(-\frac{(r-\bar{r})^{2}+(z-\bar{z})^{2}}{5 t}\right) \\
& \lesssim \frac{1}{t^{\frac{1}{2}-\frac{\alpha+\beta}{2}}} \cdot \exp \left(-\frac{(r-\bar{r})^{2}+(z-\bar{z})^{2}}{5 t}\right) \text {. }
\end{aligned}
$$

Thus combining the estimates (2.14-2.17), we conclude that whenever $(\alpha, \beta) \in \Omega_{1} \bigcup \Omega_{2}$, i.e. $\alpha, \beta$ satisfy $\alpha+\beta \leq 1, \alpha \geq-1$ and $\beta \geq-1$, there holds

$$
\begin{array}{r}
\frac{\bar{r}^{\frac{1}{2}+\beta}}{r^{\frac{1}{2}-\alpha}} \exp \left(-\frac{(r-\bar{r})^{2}+(z-\bar{z})^{2}}{4 t}\right) \cdot\left|A_{r}+\frac{r-\bar{r}}{2 t} H\left(\frac{t}{r \bar{r}}\right)\right| \\
\quad \lesssim \frac{1}{t^{\frac{1}{2}-\frac{\alpha+\beta}{2}}} \cdot \exp \left(-\frac{(r-\bar{r})^{2}+(z-\bar{z})^{2}}{5 t}\right) .
\end{array}
$$

- For $\left|A_{z}\right|$ term in the integrand (2.19). When $\bar{r}>2 r$ or $\bar{r} \leq \frac{r}{2}$, there then holds $\bar{r}+r<3|\bar{r}-r|$. If in addition $\alpha+\beta \geq-4, \alpha \geq-1$ and $\beta \geq-2$, there then exists a positive constant $\gamma$ so 
that $\max \left\{0, \frac{1}{2}-\alpha,-\frac{1}{2}-\beta,-\frac{1+\alpha+\beta}{2}\right\} \leq \gamma \leq \frac{3}{2}$. Then we deduce from Corollary 2.1 that

$$
\begin{aligned}
& \frac{\bar{r}^{\frac{1}{2}+\beta}}{r^{\frac{1}{2}-\alpha}} \exp \left(-\frac{(r-\bar{r})^{2}+(z-\bar{z})^{2}}{4 t}\right)\left(\left|A_{z}\right|+\left|\frac{r-\bar{r}}{2 t} H\left(\frac{t}{r \bar{r}}\right)\right|\right) \\
& \lesssim \frac{\bar{r}^{\frac{1}{2}+\beta}}{r^{\frac{1}{2}-\alpha}} \frac{|r-\bar{r}|+|z-\bar{z}|}{t}\left|\frac{r \bar{r}}{t}\right|^{\gamma}\left(\frac{5 t}{(r-\bar{r})^{2}+(z-\bar{z})^{2}}\right)^{\frac{1+2 \gamma+\alpha+\beta}{2}} \exp \left(-\frac{(r-\bar{r})^{2}+(z-\bar{z})^{2}}{5 t}\right) \\
& \lesssim \frac{1}{t^{\frac{1}{2}-\frac{\alpha+\beta}{2}}} \exp \left(-\frac{(r-\bar{r})^{2}+(z-\bar{z})^{2}}{5 t}\right) .
\end{aligned}
$$

And when $\frac{r}{2} \leq \bar{r} \leq 2 r$, if in addition, $-3 \leq \alpha+\beta \leq 0$, we have

$$
\begin{aligned}
& \frac{\bar{r}^{\frac{1}{2}+\beta}}{r^{\frac{1}{2}-\alpha}} \exp \left(-\frac{(r-\bar{r})^{2}+(z-\bar{z})^{2}}{4 t}\right)\left(\left|A_{z}\right|+\left|\frac{r-\bar{r}}{2 t} H\left(\frac{t}{r \bar{r}}\right)\right|\right) \\
& \lesssim \frac{\bar{r}^{\frac{1}{2}+\beta}}{r^{\frac{1}{2}-\alpha}} \frac{|r-\bar{r}|+|z-\bar{z}|}{t}\left|\frac{r \bar{r}}{t}\right|^{-\frac{\alpha+\beta}{2}}\left(\frac{5 t}{(r-\bar{r})^{2}+(z-\bar{z})^{2}}\right)^{\frac{1}{2}} \exp \left(-\frac{(r-\bar{r})^{2}+(z-\bar{z})^{2}}{5 t}\right) \\
& \lesssim \frac{1}{t^{\frac{1}{2}-\frac{\alpha+\beta}{2}}} \cdot \exp \left(-\frac{(r-\bar{r})^{2}+(z-\bar{z})^{2}}{5 t}\right) .
\end{aligned}
$$

Therefore as long as $\alpha+\beta \leq 0, \alpha \geq-1$ and $\beta \geq-2$, there holds

$$
\begin{array}{r}
\frac{\bar{r}^{\frac{1}{2}+\beta}}{r^{\frac{1}{2}-\alpha}} \exp \left(-\frac{(r-\bar{r})^{2}+(z-\bar{z})^{2}}{4 t}\right)\left(\left|A_{z}\right|+\left|\frac{r-\bar{r}}{2 t} H\left(\frac{t}{r \bar{r}}\right)\right|\right) \\
\lesssim \frac{1}{t^{\frac{1}{2}-\frac{\alpha+\beta}{2}}} \cdot \exp \left(-\frac{(r-\bar{r})^{2}+(z-\bar{z})^{2}}{5 t}\right) .
\end{array}
$$

By combining (2.24) with (2.25), we achieve

$$
\frac{\bar{r}^{\frac{1}{2}+\beta}}{r^{\frac{1}{2}-\alpha}} \exp \left(-\frac{(r-\bar{r})^{2}+(z-\bar{z})^{2}}{4 t}\right)\left(\left|A_{r}\right|+\left|A_{z}\right|\right) \lesssim \frac{1}{t^{\frac{1}{2}-\frac{\alpha+\beta}{2}}} \cdot \exp \left(-\frac{(r-\bar{r})^{2}+(z-\bar{z})^{2}}{5 t}\right),
$$

provided $\alpha+\beta \leq 0, a \geq-1$ and $\beta \geq-1$. And then (2.14) follows from (2.19) and Young's inequality in two space dimension.

(2) It follows from the proof of (2.24) that

$$
\frac{1}{r^{\frac{1}{2}-\alpha} \bar{r}^{\frac{1}{2}-\beta}}\left|H\left(\frac{t}{r \bar{r}}\right)\right| \exp \left(-\frac{(r-\bar{r})^{2}+(z-\bar{z})^{2}}{4 t}\right) \lesssim \frac{1}{t^{\frac{1}{2}-\frac{\alpha+\beta}{2}}} \exp \left(-\frac{(r-\bar{r})^{2}+(z-\bar{z})^{2}}{5 t}\right),
$$

whenever $\alpha+\beta \leq 1, \alpha \geq-1$ and $\beta \geq-1$. Then by virtue of (2.7), we get, by applying Young's inequality, that there holds (2.16).

(3) In view of (2.7), we get, by using changes of variables that

$$
\left(r^{\delta} S(t) g-r^{\delta} g\right)(r, z)=\frac{1}{4 \pi} \int_{\Omega} \Psi(r, z, \rho, \xi, t) \cdot \exp \left(-\frac{\rho^{2}+\xi^{2}}{4}\right) d \rho d \xi
$$

for all $(r, z) \in \Omega$, and where

$$
\Psi(r, z, \rho, \xi, t) \stackrel{\text { def }}{=} r^{\delta}\left(\frac{r+\sqrt{t} \rho}{r}\right)^{\frac{1}{2}} H\left(\frac{t}{r(r+\sqrt{t} \rho)}\right) g(r+\sqrt{t} \rho, z+\sqrt{t} \xi)-r^{\delta} g(r, z) .
$$


Notice that $\frac{1}{2}-\delta \in\left[0, \frac{3}{2}\right]$, applying Corollary 2.1 gives

$$
\begin{aligned}
& \left(\frac{\bar{r}}{r}\right)^{\frac{1}{2}-\delta} H\left(\frac{t}{r \bar{r}}\right) \leq C \quad \text { if } \quad \bar{r} \leq 2 r \quad \text { and } \\
& \left(\frac{\bar{r}}{r}\right)^{\frac{1}{2}-\delta} H\left(\frac{t}{r \bar{r}}\right) \leq C\left(\frac{\bar{r}}{r}\right)^{\frac{1}{2}-\delta}\left(\frac{r \bar{r}}{t}\right)^{\frac{1}{2}-\delta} \leq C\left(\frac{|\bar{r}-r|^{2}}{t}\right)^{\frac{1}{2}-\delta} \quad \text { if } \quad \bar{r}>2 r,
\end{aligned}
$$

which implies for any given $(\rho, \xi, t)$, we have

$$
\Psi(r, z, \rho, \xi, t) \lesssim\left(1+\rho^{1-2 \delta}\right) \cdot(r+\sqrt{t} \rho)^{\delta} g(r+\sqrt{t} \rho, z+\sqrt{t} \xi)-r^{\delta} g(r, z) \in L^{m}(\Omega)
$$

Moreover, noting that $H(t)=1+\mathcal{O}(t)$, as $t \rightarrow 0$, it is easy to observe that $\Psi(r, z, \rho, \xi, t) \rightarrow 0$ as $t \rightarrow 0$. Then Lebesgue dominated convergence theorem ensures that

$$
\|\Psi(\cdot, \cdot, \rho, \xi, t)\|_{L^{m}(\Omega)} \rightarrow 0, \text { as } t \rightarrow 0,
$$

from which and (2.26), another use of Lebesgue's dominated convergence theorem gives

$$
\left\|r^{\delta} S(t) g-r^{\delta} g\right\|_{L^{m}(\Omega)} \leq \frac{1}{4 \pi} \int_{\Omega}\|\Psi(\cdot, \cdot, \rho, \xi, t)\|_{L^{m}(\Omega)} \exp \left(-\frac{\rho^{2}+\xi^{2}}{4}\right) d \rho d \xi \rightarrow 0, \text { as } t \rightarrow 0 .
$$

This completes the proof of the proposition.

\section{LOCAL EXISTENCE OF SOlUtions to (1.1) IN CRITICAL SPACES}

The purpose of this section is to investigate the local existence and uniqueness of the mild solutions to (1.4) in the spirit of $[5,6]$. In view of $(2.7)$, we rewrite the systems (1.4) as

$$
\begin{cases}\omega^{\theta}(t)=S(t) \omega_{0}^{\theta}-\int_{0}^{t} S(t-s)\left(\operatorname{div}_{*}\left(\widetilde{u}(s) \omega^{\theta}(s)\right)-\frac{\partial_{z}\left|u^{\theta}(s)\right|^{2}}{r}\right) d s, & t>0, \\ u^{\theta}(t)=S(t) u_{0}^{\theta}-\int_{0}^{t} S(t-s)\left(\operatorname{div}_{*}\left(\widetilde{u}(s) u^{\theta}(s)\right)-\frac{2 u^{\theta}(s) u^{r}(s)}{r}\right) d s, & t>0,\end{cases}
$$

Now we present the proof of Theorem 1.1.

Proof of Theorem 1.1. The main idea to prove Theorem 1.1 is to apply fixed point argument for the integral formulation (3.1). Toward this, for any $T>0$, we introduce the functional space

$$
\begin{aligned}
\left.\left.\left.X_{T} \stackrel{\text { def }}{=}\left\{\left(\omega^{\theta}, u^{\theta}\right) \in C(] 0, T\right] ; L^{\frac{4}{3}}(\Omega)\right) \times C(] 0, T\right] ; L^{4}(\Omega)\right) \mid \\
\left.\left.\left.r^{-\frac{3}{10}} u^{\theta} \in C(] 0, T\right] ; L^{2}(\Omega)\right) \text { and }\left\|\left(\omega^{\theta}, u^{\theta}\right)\right\|_{X_{T}}<\infty\right\},
\end{aligned}
$$

where

$$
\left\|\left(\omega^{\theta}, u^{\theta}\right)\right\|_{X_{T}} \stackrel{\text { def }}{=} \sup _{0<t \leq T}\left(t^{\frac{1}{4}}\left\|\omega^{\theta}(t)\right\|_{L^{\frac{4}{3}}(\Omega)}+t^{\frac{1}{4}}\left\|u^{\theta}(t)\right\|_{L^{4}(\Omega)}+t^{\frac{3}{20}}\left\|r^{-\frac{3}{10}} u^{\theta}(t)\right\|_{L^{2}(\Omega)}\right) .
$$

For convenience, sometimes we may abuse the notation $\left\|\omega^{\theta}\right\|_{X_{T}}=\sup _{0<t \leq T} t^{\frac{1}{4}}\left\|\omega^{\theta}\right\|_{L^{\frac{4}{3}}(\Omega)},\left\|u^{\theta}\right\|_{X_{T}}=$ $\sup _{0<t \leq T}\left(t^{\frac{1}{4}}\left\|u^{\theta}(t)\right\|_{L^{4}(\Omega)}+t^{\frac{3}{20}}\left\|r^{-\frac{3}{10}} u^{\theta}(t)\right\|_{L^{2}(\Omega)}\right)$, and $\omega^{\theta} \in X_{T}$ (resp. $u^{\theta} \in X_{T}$ ) means that $\left\|\omega^{\theta}\right\|_{X_{T}}<\infty$ (resp. $\left.\left\|u^{\theta}\right\|_{X_{T}}<\infty\right)$.

- The estimate of $\omega^{\theta}$ term

In view of (2.17), $S(t) \omega_{0}^{\theta} \in X_{T}$ for any $T>0$, and there exists a universal constant $C_{1}>0$ such that for any $T>0$, we have

$$
\sup _{0<t \leq T} t^{\frac{1}{4}}\left\|S(t) \omega_{0}^{\theta}\right\|_{L^{\frac{4}{3}(\Omega)}} \leq C_{1}\left\|\omega_{0}^{\theta}\right\|_{L^{1}(\Omega)} .
$$


On the other hand, since $L^{1}(\Omega) \cap L^{\frac{4}{3}}(\Omega)$ is dense in $L^{1}(\Omega)$. For any $\varepsilon>0$, there exists $\widetilde{\omega}_{0}^{\theta} \in L^{1}(\Omega) \bigcap L^{\frac{4}{3}}(\Omega)$ satisfying $\left\|\widetilde{\omega}_{0}^{\theta}-\omega_{0}^{\theta}\right\|_{L^{1}(\Omega)}<\varepsilon$. Then it follows from (2.17) that

$$
\begin{aligned}
t^{\frac{1}{4}}\left\|S(t) \omega_{0}^{\theta}\right\|_{L^{\frac{4}{3}}(\Omega)} & \leq t^{\frac{1}{4}}\left\|S(t)\left(\omega_{0}^{\theta}-\widetilde{\omega}_{0}^{\theta}\right)\right\|_{L^{\frac{4}{3}}(\Omega)}+t^{\frac{1}{4}}\left\|S(t) \widetilde{\omega}_{0}^{\theta}\right\|_{L^{\frac{4}{3}}(\Omega)} \\
& \leq C\left(\left\|\widetilde{\omega}_{0}^{\theta}-\omega_{0}^{\theta}\right\|_{L^{1}(\Omega)}+t^{\frac{1}{4}}\left\|\widetilde{\omega}_{0}^{\theta}\right\|_{L^{\frac{4}{3}}(\Omega)}\right) \\
& \leq C\left(\varepsilon+t^{\frac{1}{4}}\left\|\widetilde{\omega}_{0}^{\theta}\right\|_{L^{\frac{4}{3}}(\Omega)}\right)
\end{aligned}
$$

which implies

$$
\lim _{t \rightarrow 0} t^{\frac{1}{4}}\left\|S(t) \omega_{0}^{\theta}\right\|_{L^{\frac{4}{3}(\Omega)}}=0
$$

Let us denote $\widetilde{u}\left(\omega^{\theta}\right)(t)$ be velocity field determined by the vorticity $\omega^{\theta} e_{\theta}$ via the axisymmetric Biot-Savart law $(2.2)$. Given $\left(\omega_{1}^{\theta}, u_{1}^{\theta}\right),\left(\omega_{2}^{\theta}, u_{2}^{\theta}\right) \in X_{T}$, for any $t \in[0, T]$, we define the mapping $\mathcal{F}^{\omega}$ on $X_{T} \times X_{T}$ by

$$
\mathcal{F}^{\omega}\left(\left(\omega_{1}^{\theta}, u_{1}^{\theta}\right),\left(\omega_{2}^{\theta}, u_{2}^{\theta}\right)\right)(t) \stackrel{\text { def }}{=} \int_{0}^{t} S(t-s) \cdot\left(\operatorname{div} *\left(\widetilde{u}\left(\omega_{1}^{\theta}\right)(s) \omega_{2}^{\theta}(s)\right)-\frac{\partial_{z}\left(u_{1}^{\theta}(s) u_{2}^{\theta}(s)\right)}{r}\right) d s
$$

Then for any $p \in\left[1, \frac{10}{7}\left[\right.\right.$, we deduce from (2.14) (with $\left.\alpha=0, \beta=-\frac{2}{5}\right),(2.15)$ and (2.4) that for any $t \in] 0, T]$,

$$
\begin{aligned}
& t^{1-\frac{1}{p}}\left\|\mathcal{F}^{\omega}\left(\left(\omega_{1}, u_{1}^{\theta}\right),\left(\omega_{2}, u_{2}^{\theta}\right)\right)(t)\right\|_{L^{p}(\Omega)} \\
& \leq C t^{1-\frac{1}{p}} \int_{0}^{t} \frac{\left\|\widetilde{u}\left(\omega_{1}^{\theta}\right)(s) \cdot \omega_{2}^{\theta}(s)\right\|_{L^{1}(\Omega)}}{(t-s)^{\frac{3}{2}-\frac{1}{p}}}+\frac{\left\|r^{-\frac{3}{5}} u_{1}^{\theta}(s) u_{2}^{\theta}(s)\right\|_{L^{1}(\Omega)}}{(t-s)^{\frac{3}{2}-\frac{1}{p}+\frac{1}{5}}} d s \\
& \leq C t^{1-\frac{1}{p}} \int_{0}^{t} \frac{\left\|\widetilde{u}\left(\omega_{1}^{\theta}\right)(s)\right\|_{L^{4}(\Omega)}\left\|\omega_{2}^{\theta}(s)\right\|_{L^{\frac{4}{3}}(\Omega)}}{(t-s)^{\frac{3}{2}-\frac{1}{p}}}+\frac{\left\|r^{-\frac{3}{10}} u_{1}^{\theta}(s)\right\|_{L^{2}(\Omega)}\left\|r^{-\frac{3}{10}} u_{2}^{\theta}(s)\right\|_{L^{2}(\Omega)}}{(t-s)^{\frac{3}{2}-\frac{1}{p}+\frac{1}{5}}} d s \\
& \leq C t^{1-\frac{1}{p}} \int_{0}^{t} \frac{\left\|\omega_{1}^{\theta}\right\|_{X_{T}}\left\|\omega_{2}^{\theta}\right\|_{X_{T}}}{(t-s)^{\frac{3}{2}-\frac{1}{p}} \cdot s^{\frac{1}{2}}}+\frac{\left\|u_{1}^{\theta}\right\|_{X_{T}}\left\|u_{2}^{\theta}\right\|_{X_{T}}}{(t-s)^{\frac{17}{10}-\frac{1}{p}} \cdot s^{\frac{3}{10}}} d s \\
& \leq A_{p}^{\omega}\left(\left\|\omega_{1}^{\theta}\right\|_{X_{T}}\left\|\omega_{2}^{\theta}\right\|_{X_{T}}+\left\|u_{1}^{\theta}\right\|_{X_{T}}\left\|u_{2}^{\theta}\right\|_{X_{T}}\right) .
\end{aligned}
$$

\section{- The estimate of $u^{\theta}$ terms}

Thanks to (2.16) (with $\alpha=-\frac{3}{10}, \beta=\frac{13}{10}$ ), by a similar derivation of (3.4), (3.5), we get

$$
\begin{aligned}
& \sup _{0<t \leq T}\left(t^{\frac{1}{4}}\left\|S(t) u_{0}^{\theta}\right\|_{L^{4}(\Omega)}+t^{\frac{3}{20}}\left\|r^{-\frac{3}{10}} S(t) u_{0}^{\theta}\right\|_{L^{2}(\Omega)}\right) \leq C_{1}\left(\left\|u_{0}^{\theta}\right\|_{L^{2}(\Omega)}+\left\|r^{-\frac{3}{10}} u_{0}^{\theta}\right\|_{L^{\frac{20}{13}}(\Omega)}\right) \\
& \quad \text { and } \lim _{t \rightarrow 0}\left(t^{\frac{1}{4}}\left\|S(t) u_{0}^{\theta}\right\|_{L^{4}(\Omega)}+t^{\frac{3}{20}}\left\|r^{-\frac{3}{10}} S(t) u_{0}^{\theta}\right\|_{L^{2}(\Omega)}\right)=0
\end{aligned}
$$

Given $\left(\omega_{1}^{\theta}, u_{1}^{\theta}\right),\left(\omega_{2}^{\theta}, u_{2}^{\theta}\right) \in X_{T}$, for any $t \in[0, T]$, we define the mapping $\mathcal{F}^{u}$ on $X_{T} \times X_{T}$ by

$$
\mathcal{F}^{u}\left(\left(\omega_{1}, u_{1}^{\theta}\right),\left(\omega_{2}, u_{2}^{\theta}\right)\right)(t) \stackrel{\text { def }}{=} \int_{0}^{t} S(t-s)\left(\operatorname{div}_{*}\left(\widetilde{u}\left(\omega_{1}^{\theta}\right)(s) \cdot u_{2}^{\theta}(s)\right)-\frac{2 u^{r}\left(\omega_{1}^{\theta}\right)(s) \cdot u_{2}^{\theta}(s)}{r}\right) d s
$$


Then for any $q \in[2, \infty[$, we deduce from $(2.15),(2.17)$ and (2.4) that for any $t \in] 0, T]$

$$
\begin{aligned}
& t^{\frac{1}{2}-\frac{1}{q}}\left\|\mathcal{F}^{u}\left(\left(\omega_{1}, u_{1}^{\theta}\right),\left(\omega_{2}, u_{2}^{\theta}\right)\right)(t)\right\|_{L^{q}(\Omega)} \\
& \leq t^{\frac{1}{2}-\frac{1}{q}} \int_{0}^{t} \frac{C}{(t-s)^{1-\frac{1}{q}}}\left\|\widetilde{u}\left(\omega_{1}^{\theta}\right)(s) \cdot u_{2}^{\theta}(s)\right\|_{L^{2}(\Omega)} d s \\
& \leq t^{\frac{1}{2}-\frac{1}{q}} \int_{0}^{t} \frac{C}{(t-s)^{1-\frac{1}{q}}}\left\|\widetilde{u}\left(\omega_{1}^{\theta}\right)(s)\right\|_{L^{4}(\Omega)}\left\|u_{2}^{\theta}(s)\right\|_{L^{4}(\Omega)} d s \\
& \leq t^{\frac{1}{2}-\frac{1}{q}} \int_{0}^{t} \frac{C}{(t-s)^{1-\frac{1}{q}}}\left\|\omega_{1}^{\theta}(s)\right\|_{L^{\frac{4}{3}}(\Omega)}\left\|u_{2}^{\theta}(s)\right\|_{L^{4}(\Omega)} d s \\
& \leq t^{\frac{1}{2}-\frac{1}{q}} \int_{0}^{t} \frac{C}{(t-s)^{1-\frac{1}{q}}} \frac{\left\|\omega_{1}^{\theta}\right\| X_{t}\left\|u_{2}^{\theta}\right\|_{X_{t}}}{s^{\frac{1}{2}}} d s \leq A_{q}^{u}\left\|\omega_{1}^{\theta}\right\|_{X_{T}}\left\|u_{2}^{\theta}\right\|_{X_{T}} .
\end{aligned}
$$

Along the same line, for any $\kappa \in\left[\frac{20}{13}, 4\left[\right.\right.$, we deduce from (2.14), (2.16) (with $\alpha=-\frac{3}{10}, \beta=$ $\left.\frac{3}{10}\right)$ and (2.4) that for any $\left.\left.t \in\right] 0, T\right]$

$$
\begin{aligned}
& t^{\frac{13}{20}-\frac{1}{\kappa}}\left\|r^{-\frac{3}{10}} \mathcal{F}^{u}\left(\left(\omega_{1}, u_{1}^{\theta}\right),\left(\omega_{2}, u_{2}^{\theta}\right)\right)(t)\right\|_{L^{\kappa}(\Omega)} \\
& \leq t^{\frac{13}{20}-\frac{1}{\kappa}} \int_{0}^{t} \frac{C}{(t-s)^{\frac{5}{4}-\frac{1}{\kappa}}}\left\|\widetilde{u}\left(\omega_{1}^{\theta}\right)(s) \cdot r^{-\frac{3}{10}} u_{2}^{\theta}(s)\right\|_{L^{\frac{4}{3}}(\Omega)} d s \\
& \leq t^{\frac{13}{20}-\frac{1}{\kappa}} \int_{0}^{t} \frac{C}{(t-s)^{\frac{5}{4}-\frac{1}{\kappa}}}\left\|\widetilde{u}\left(\omega_{1}^{\theta}\right)(s)\right\|_{L^{4}(\Omega)}\left\|r^{-\frac{3}{10}} u_{2}^{\theta}(s)\right\|_{L^{2}(\Omega)} d s \\
& \leq t^{\frac{13}{20}-\frac{1}{\kappa}} \int_{0}^{t} \frac{C}{(t-s)^{\frac{5}{4}-\frac{1}{\kappa}}} \frac{\left\|\omega_{1}^{\theta}\right\|_{X_{t}}\left\|u_{2}^{\theta}\right\|_{X_{t}}}{s^{\frac{2}{5}}} d s \leq B_{k}^{u}\left\|\omega_{1}^{\theta}\right\|_{X_{T}}\left\|u_{2}^{\theta}\right\|_{X_{T}} .
\end{aligned}
$$

- Fixed point argument

For $\left(\omega_{1}^{\theta}, u_{1}^{\theta}\right),\left(\omega_{2}^{\theta}, u_{2}^{\theta}\right) \in X_{T}$, we consider the following bilinear map

$$
\mathcal{F}\left(\left(\omega_{1}, u_{1}^{\theta}\right),\left(\omega_{2}, u_{2}^{\theta}\right)\right) \stackrel{\text { def }}{=}\left(\mathcal{F}^{\omega}, \mathcal{F}^{u}\right)\left(\left(\omega_{1}, u_{1}^{\theta}\right),\left(\omega_{2}, u_{2}^{\theta}\right)\right),
$$

with $\mathcal{F}^{\omega}, \mathcal{F}^{u}$ given by (3.6) and (3.9) respectively.

By virtue of (3.5) and (3.8), for any $\omega_{0}^{\theta} \in L^{1}(\Omega), u_{0}^{\theta} \in L^{2}(\Omega)$ with $r^{-\frac{3}{10}} u_{0}^{\theta} \in L^{\frac{20}{13}}(\Omega)$, there exists a positive time $T$ such that

$$
\begin{aligned}
E L(T) & \stackrel{\text { def }}{=} \sup _{t \in[0, T]}\left(t^{\frac{1}{4}}\left\|S(t) \omega_{0}^{\theta}\right\|_{L^{\frac{4}{3}(\Omega)}}+t^{\frac{1}{4}}\left\|S(t) u_{0}^{\theta}\right\|_{L^{4}(\Omega)}+t^{\frac{3}{20}}\left\|r^{-\frac{3}{10}} S(t) u_{0}^{\theta}\right\|_{L^{2}(\Omega)}\right) \\
& \leq \frac{1}{4\left(A_{4 / 3}^{\omega}+A_{4}^{u}+B_{2}^{u}\right)},
\end{aligned}
$$

where the constants $A_{4 / 3}^{\omega}, A_{4}^{u}$ and $B_{2}^{u}$ are determined by (3.7), (3.10) and (3.11) respectively. Then we deduce from Lemma 2.2 that (3.1) has a unique solution $\left(\omega^{\theta}, u^{\theta}\right)$ in $X_{T}$. Furthermore, by virtue of (3.5) and (3.8), for any $\varepsilon>0$, there exists $T_{\varepsilon}>0$ so that

$$
E L\left(T_{\varepsilon}\right) \leq \varepsilon
$$

Then Lemma 2.2 ensures that

$$
\left\|\left(\omega^{\theta}, u^{\theta}\right)\right\|_{X_{T_{\varepsilon}}} \leq 2 \varepsilon
$$


which implies that

$$
\lim _{T \rightarrow 0}\left\|\left(\omega^{\theta}, u^{\theta}\right)\right\|_{X_{T}}=0 .
$$

Futhermore, for any $T>0$, it follows from (3.4) and (3.8) that

$$
E L(T) \leq C_{1}\left(\left\|\omega_{0}^{\theta}\right\|_{L^{1}(\Omega)}+\left\|u_{0}^{\theta}\right\|_{L^{2}(\Omega)}+\left\|r^{-\frac{3}{10}} u_{0}^{\theta}\right\|_{L^{20}(\Omega)}\right) \leq \frac{1}{4\left(A_{4 / 3}^{\omega}+A_{4}^{u}+B_{2}^{u}\right)},
$$

provided that $c$ in (1.9) satisfying $c \leq \frac{1}{4 C_{1}\left(A_{4 / 3}^{\omega}+A_{4}^{u}+B_{2}^{u}\right)}$. This together with Lemma 2.2 shows that (3.1) has a unique global solution in $X_{\infty}$.

- Behavior near $t=0$

Let us now turn to the estimate (1.8). Let $\left(\omega^{\theta}, u^{\theta}\right)$ be the unique solution of $(3.1)$ on $[0, T]$ obtained in the previous one step, we denote

$$
J_{p, q, \kappa}(T) \stackrel{\text { def }}{=} \sup _{0 \leq t \leq T}\left(t^{1-\frac{1}{p}}\left\|S(t) \omega_{0}^{\theta}\right\|_{L^{p}(\Omega)}+t^{\frac{1}{2}-\frac{1}{q}}\left\|S(t) u_{0}^{\theta}\right\|_{L^{q}(\Omega)}+t^{\frac{13}{20}-\frac{1}{\kappa}}\left\|r^{-\frac{3}{10}} S(t) u_{0}^{\theta}\right\|_{L^{\kappa}(\Omega)} .\right.
$$

Along the same line to the proof of (3.5), it is easy to observe that

$$
\left.\left.\left.\left.\left.\left.\lim _{T \rightarrow 0} J_{p, q, \kappa}(T)=0, \quad \forall p \in\right] 1, \infty\right], q \in\right] 2, \infty\right], \kappa \in\right] 20 / 13, \infty\right] .
$$

While it follows from the proof of (3.7), (3.10) and (3.11) that

$$
\begin{aligned}
\left\|\omega^{\theta}(t)-S(t) \omega_{0}^{\theta}\right\|_{L^{1}(\Omega)} & \leq A_{p}^{\omega}\left(\left\|\omega^{\theta}\right\|_{X_{t}}^{2}+\left\|u^{\theta}\right\|_{X_{t}}^{2}\right), \\
\left\|u^{\theta}(t)-S(t) u_{0}^{\theta}\right\|_{L^{2}(\Omega)} & \leq A_{2}^{u}\left\|\omega^{\theta}\right\|_{X_{t}}\left\|u^{\theta}\right\|_{X_{t}}, \\
\left\|r^{-\frac{3}{10}} u^{\theta}(t)-r^{-\frac{3}{10}} S(t) u_{0}^{\theta}\right\|_{L^{\frac{20}{13}(\Omega)}} & \leq B_{\frac{20}{13}}^{u}\left\|\omega^{\theta}\right\|_{X_{t}}\left\|u^{\theta}\right\|_{X_{t}},
\end{aligned}
$$

from which, (2.18) and (3.14), we infer

$$
\lim _{t \rightarrow 0}\left(\left\|\omega^{\theta}(t)-\omega_{0}^{\theta}\right\|_{L^{1}(\Omega)}+\left\|u^{\theta}(t)-u_{0}^{\theta}\right\|_{L^{2}(\Omega)}+\left\|r^{-\frac{3}{10}} u^{\theta}(t)-r^{-\frac{3}{10}} u_{0}^{\theta}\right\|_{L^{\frac{20}{13}}(\Omega)}\right)=0 .
$$

Whereas for any $t_{0}>0$, we get, by using a similar derivation of (3.7), that

$$
\begin{aligned}
& \left\|\int_{t_{0}}^{t} S(t-s)\left(\operatorname{div}_{*}\left(\widetilde{u}(s) \omega^{\theta}(s)\right)+\frac{2 u^{\theta}(s) \omega^{r}(s)}{r}\right) d s\right\|_{L^{1}(\Omega)} \\
& \quad \leq C \int_{t_{0}}^{t} \frac{\left\|\omega_{1}^{\theta}\right\|_{X_{T}}\left\|\omega_{2}^{\theta}\right\|_{X_{T}}}{(t-s)^{\frac{1}{2}} \cdot s^{\frac{1}{2}}}+\frac{\left\|u_{1}^{\theta}\right\|_{X_{T}}\left\|u_{2}^{\theta}\right\|_{X_{T}}}{(t-s)^{\frac{7}{10}} \cdot s^{\frac{3}{10}}} d s \\
& \quad \leq C\left(t_{0}^{-\frac{1}{2}}\left(t-t_{0}\right)^{\frac{1}{2}}+t_{0}^{-\frac{3}{10}}\left(t-t_{0}\right)^{\frac{3}{10}}\right)\left(\left\|\omega^{\theta}\right\|_{X_{t}}^{2}+\left\|u^{\theta}\right\|_{X_{t}}\left\|\omega^{r}\right\|_{X_{t}}\right) .
\end{aligned}
$$

Hence by virtue of the expression (3.1), we deduce that

$$
\lim _{t \rightarrow t_{0}}\left\|\omega^{\theta}(t)-\omega^{\theta}\left(t_{0}\right)\right\|_{L^{1}(\Omega)}=0 .
$$

Exactly along the same line, we can prove that

$$
\lim _{t \rightarrow t_{0}}\left(\left\|u^{\theta}(t)-u^{\theta}\left(t_{0}\right)\right\|_{L^{2}(\Omega)}+\left\|r^{-\frac{3}{10}} u^{\theta}(t)-r^{-\frac{3}{10}} u^{\theta}\left(t_{0}\right)\right\|_{L^{\frac{20}{13}}(\Omega)}\right)=0,
$$

This together with (3.16) and (3.17) ensures that

$$
\omega \in C\left([0, T] ; L^{1}(\Omega)\right), u^{\theta} \in C\left([0, T] ; L^{2}(\Omega)\right) \text { and } r^{-\frac{3}{10}} u^{\theta} \in C\left([0, T] ; L^{\frac{20}{13}}(\Omega)\right) .
$$

For any $q \in] 2, \infty]$, by using (3.10), (3.14) and (3.15), we deduce that $M_{q}(T)$ are bounded, and $M_{q}(T) \rightarrow 0$ as $T \rightarrow 0$. 
For the estimate of $N_{\kappa}(T)$ and $L_{p}(T)$, we shall use a bootstrap argument. Indeed to estimate $N_{\kappa}(T)$, we get, by a similar derivation of (3.11), that

$$
\begin{aligned}
& t^{\frac{13}{20}-\frac{1}{\kappa}}\left\|r^{-\frac{3}{10}} \mathcal{F}^{u}\left(\left(\omega, u^{\theta}\right),\left(\omega, u^{\theta}\right)\right)(t)\right\|_{L^{\kappa}(\Omega)} \\
& \leq t^{\frac{13}{20}-\frac{1}{\kappa}} \int_{0}^{t} \frac{C}{(t-s)^{\frac{3}{4}+\frac{1}{\kappa_{1}}-\frac{1}{\kappa}}}\left\|\widetilde{u}\left(\omega^{\theta}\right)(s) \cdot r^{-\frac{3}{10}} u^{\theta}(s)\right\|_{L^{\frac{4 \kappa_{1}}{4+\kappa_{1}}(\Omega)}} d s
\end{aligned}
$$

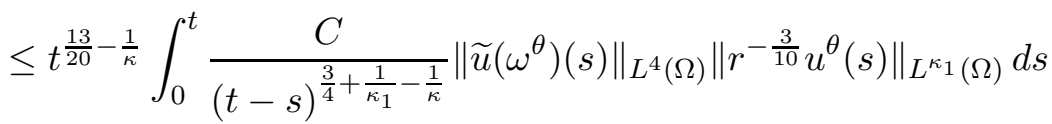

$$
\begin{aligned}
& \leq t^{\frac{13}{20}-\frac{1}{\kappa}} \int_{0}^{t} \frac{C}{(t-s)^{\frac{3}{4}+\frac{1}{\kappa_{1}}-\frac{1}{\kappa}}} \frac{\left\|\omega^{\theta}\right\|_{X_{t}} N_{\kappa_{1}}(T)}{s^{\frac{9}{10}-\frac{1}{\kappa_{1}}}} d s \leq B_{\kappa, \kappa_{1}}^{u}\left\|\omega^{\theta}\right\|_{X_{T}} N_{\kappa_{1}}(T),
\end{aligned}
$$

where the exponents $\left.\left.\kappa \in] 20 / 13, \infty], \kappa_{1} \in\right] 20 / 13, \infty\right]$ satisfying

$$
\frac{4 \kappa_{1}}{4+\kappa_{1}} \leq \kappa \quad \text { and } \quad \frac{3}{4}+\frac{1}{\kappa_{1}}-\frac{1}{\kappa}<1
$$

Meanwhile it follows from (3.1) and (3.19) that

$$
N_{\kappa}(T) \leq J_{p, q, \kappa}(T)+B_{\kappa, \kappa_{1}}^{u}\left\|\omega^{\theta}\right\|_{X_{T}} N_{\kappa_{1}}(T) .
$$

Note that $J_{p, q, \kappa}(T),\left\|\omega^{\theta}\right\|_{X_{T}}, N_{2}(T) \rightarrow 0$ as $T \rightarrow 0$, by taking $\kappa_{1}=2$ in (3.21), it follows from (3.20) that for any $\kappa \in] 20 / 13,4[$, we have

$$
N_{\kappa}(T) \quad \text { are bounded and } \quad N_{\kappa}(T) \rightarrow 0 \quad \text { as } T \rightarrow 0 .
$$

Next, taking $\kappa_{1}=3$ in (3.21), we deduce from (3.20) that (3.22) holds for any $\kappa \in[4,12$ [. Along the same line, taking $\kappa_{1}=10$ in $(3.21)$ ensures $(3.22)$ for any $\kappa \in[12, \infty]$. Hence we prove that $(3.22)$ holds for any $\kappa \in] 20 / 13, \infty]$.

To handle $L_{p}(T)$, we get, by a similar derivation of (3.7), that but here we need to split the integral area in two parts,

$$
\begin{aligned}
& t^{1-\frac{1}{p}}\left\|\mathcal{F}^{\omega}\left(\left(\omega, u^{\theta}\right),\left(\omega, u^{\theta}\right)\right)(t)\right\|_{L^{p}(\Omega)} \\
& \leq C t^{1-\frac{1}{p}} \int_{0}^{\frac{t}{2}} \frac{\left(s^{\frac{1}{4}}\left\|\omega^{\theta}(s)\right\|_{L^{\frac{4}{3}}(\Omega)}\right)^{2}}{(t-s)^{\frac{3}{2}-\frac{1}{p}} s^{\frac{1}{2}}}+\frac{\left(s^{\frac{3}{20}}\left\|r^{-\frac{3}{10}} u^{\theta}(s)\right\|_{L^{2}(\Omega)}\right)^{2}}{(t-s)^{\frac{3}{2}-\frac{1}{p}+\frac{1}{5}} s^{\frac{3}{10}}} d s \\
& \quad+C t^{1-\frac{1}{p}} \int_{\frac{t}{2}}^{t}\left(\frac{\left(s^{1-\frac{1}{p_{1}}}\left\|\omega^{\theta}(s)\right\|_{L^{p_{1}}(\Omega)}\right)^{2}}{(t-s)^{\frac{2}{p_{1}}-\frac{1}{p}} s^{2-\frac{2}{p_{1}}}}+\frac{\left(s^{\frac{13}{20}-\frac{1}{2 p}}\left\|r^{-\frac{3}{10}} u^{\theta}(s)\right\|_{L^{2 p}(\Omega)}\right)^{2}}{(t-s)^{\frac{7}{10}} s^{\frac{13}{10}-\frac{1}{p}}}\right) d s \\
& \leq A_{p, p_{1}, p_{2}}^{\omega}\left(\left\|\omega^{\theta}\right\|_{X_{T}}^{2}+\left\|u^{\theta}\right\|_{X_{T}}^{2}+L_{p_{1}}^{2}(T)+N_{2 p}^{2}(T)\right) .
\end{aligned}
$$

where the exponents $\left.\left.p, p_{1} \in\right] 1, \infty\right]$ satisfying

$$
\frac{1}{2} \leq \frac{2}{p_{1}}-\frac{1}{p}<1
$$

Then we deduce from (3.1) and (3.23) that

$$
L_{p}(T) \leq J_{p, q, \kappa}(T)+A_{p, p_{1}, p_{2}}^{\omega}\left(\left\|\omega^{\theta}\right\|_{X_{T}}^{2}+\left\|u^{\theta}\right\|_{X_{T}}^{2}+L_{p_{1}}^{2}(T)+N_{2 p}^{2}(T)\right) .
$$

Note that $J_{p, q, \kappa}(T), L_{\frac{4}{3}}=\left\|\omega^{\theta}\right\|_{X_{T}},\left\|u^{\theta}\right\|_{X_{T}}, N_{2 p}(T) \rightarrow 0$ as $T \rightarrow 0$, by taking $p_{1}=\frac{4}{3}$ in (3.25), we deduce from (3.24), that

$$
L_{p}(T) \text { are bounded and } L_{p}(T) \rightarrow 0 \quad \text { as } T \rightarrow 0
$$


for any $p \in] 1,2\left[\right.$. Next taking $p_{1}=\frac{5}{3}$ in (3.25) ensures that (3.26) holds for any $p \in[2,5[$. Similarly, taking $p_{1}=\frac{5}{2}$ in (3.25) implies that (3.26) holds for any $p \in[5, \infty]$. Thus $(3.26)$ holds for any $p \in] 1, \infty]$. This completes the proof of Theorem 1.1.

Let $L_{p}(T), M_{q}(T), N_{\kappa}(T)$ be given by (1.7), for any $s \in \mathbb{N}^{+}$, we shall denote

$$
\begin{aligned}
& L_{p_{1}, p_{2}}(T) \stackrel{\text { def }}{=} \sup _{p_{1} \leq p \leq p_{2}} L_{p}(T), \quad M_{q_{1}, q_{2}}(T) \stackrel{\text { def }}{=} \sup _{q_{1} \leq q \leq q_{2}} M_{q}(T), \\
& N_{\kappa_{1}, \kappa_{2}}(T) \stackrel{\text { def }}{=} \sup _{\kappa_{1} \leq \kappa \leq \kappa_{2}} N_{\kappa}(T) \quad \text { and } \quad L_{p_{1}, p_{2}}^{s}(T) \stackrel{\text { def }}{=} L_{p_{1}, p_{2}}(T)+\left|L_{p_{1}, p_{2}}(T)\right|^{s}, \\
& M_{q_{1}, q_{2}}^{s}(T) \stackrel{\text { def }}{=} M_{q_{1}, q_{2}}(T)+\left|M_{q_{1}, q_{2}}(T)\right|^{s}, \quad N_{\kappa_{1}, \kappa_{2}}^{s}(T) \stackrel{\text { def }}{=} N_{\kappa_{1}, \kappa_{2}}(T)+\left|N_{\kappa_{1}, \kappa_{2}}(T)\right|^{s} .
\end{aligned}
$$

For later use, we state the following result.

Corollary 3.1. For any $0 \leq \gamma \leq \delta \leq 1,1 \leq p \leq \infty, 1 \leq q_{2} \leq q_{1} \leq \infty$, under the assumptions of Theorem 1.1, if we assume moreover that $r^{-\gamma} u_{0}^{\theta} \in L^{q_{2}}(\Omega)$, then there hold

$$
\begin{aligned}
& \left\|r^{-\delta} u^{\theta}(t)\right\|_{L^{q_{1}}(\Omega)} \lesssim \frac{L_{\frac{20}{17}, 2}(T) N_{\frac{20}{13}, 20}(T)}{t^{\frac{1}{2}+\frac{\delta}{2}-\frac{1}{q_{1}}}}+\frac{\left\|r^{-\gamma} u_{0}^{\theta}\right\|_{L^{q_{2}}(\Omega)}}{t^{\frac{\delta-\gamma}{2}+\frac{1}{q_{2}}-\frac{1}{q_{1}}}}, \quad \forall t \in[0, T], \\
& \left\|r^{-\delta} \omega^{\theta}(t)\right\|_{L^{p}(\Omega)} \lesssim \frac{L_{1, \frac{20}{3}}^{5}(T)+M_{2}^{3}(T)+N_{\frac{20}{13}, 20}^{5}(T)}{t^{1+\frac{\delta}{2}-\frac{1}{p}}}, \quad \forall t \in[0, T] .
\end{aligned}
$$

Proof. When $q_{1} \in[1,10]$, in view of $(3.1)$, we get, by applying Proposition 2.2 and then Lemma 2.4, that

$$
\begin{gathered}
\left\|r^{-\delta} u^{\theta}(t)\right\|_{L^{q_{1}}(\Omega)} \lesssim \frac{\left\|r^{-\gamma} u_{0}^{\theta}\right\|_{L^{q_{2}}(\Omega)}}{t^{\frac{\delta-\gamma}{2}+\frac{1}{q_{2}}-\frac{1}{q_{1}}}}+\int_{0}^{\frac{t}{2}} \frac{\|\widetilde{u}(s)\|_{L^{\frac{20}{7}}(\Omega)}\left\|r^{-\frac{3}{10}} u^{\theta}(s)\right\|_{L^{\frac{20}{13}}(\Omega)}}{(t-s)^{\frac{27}{20}+\frac{\delta}{2}-\frac{1}{q_{1}}}} d s \\
\quad+\int_{\frac{t}{2}}^{t} \frac{\|\widetilde{u}(s)\|_{L^{\frac{20}{7} q_{1}}(\Omega)}\left\|r^{-\frac{3}{10}} u^{\theta}(s)\right\|_{L^{\frac{20}{13} q_{1}}(\Omega)}}{(t-s)^{\frac{7}{20}+\frac{\delta}{2}}} d s \\
\lesssim \frac{\left\|r^{-\gamma} u_{0}^{\theta}\right\|_{L^{q_{2}}(\Omega)}}{t^{\frac{\delta-\gamma}{2}+\frac{1}{q_{2}}-\frac{1}{q_{1}}}}+\int_{0}^{\frac{t}{2}} \frac{L_{\frac{20}{17}}(T) N_{\frac{20}{13}}(T)}{(t-s)^{\frac{27}{20}+\frac{\delta}{2}-\frac{1}{q_{1}}} s^{\frac{3}{20}}} d s+\int_{\frac{t}{2}}^{t} \frac{L_{\frac{20 q_{1}}{10 q_{1}+7}}(T) N_{\frac{20}{13} q_{1}}(T)}{(t-s)^{\frac{7}{20}+\frac{\delta}{2}} s^{\frac{23}{20}-\frac{1}{q_{1}}}} d s \\
\lesssim \frac{\left\|r^{-\gamma} u_{0}^{\theta}\right\|_{L^{q_{2}}(\Omega)}}{t^{\frac{\delta-\gamma}{2}+\frac{1}{q_{2}}-\frac{1}{q_{1}}}}+\frac{L_{\frac{20}{17}}(T) N_{\frac{20}{13}}(T)+L_{\frac{20 q_{1}}{10 q_{1}+7}}(T) N_{\frac{20}{13} q_{1}}(T)}{q_{1}}
\end{gathered}
$$

which yields $(3.28)$ for $q_{1} \in[1,10]$.

When $\left.\left.q_{1} \in\right] 10, \infty\right]$, we get, by a similar derivation, that

$$
\begin{gathered}
\left\|r^{-\delta} u^{\theta}(t)\right\|_{L^{q_{1}}(\Omega)} \lesssim \frac{\left\|r^{-\gamma} u_{0}^{\theta}\right\|_{L^{q_{2}}(\Omega)}}{t^{\frac{\delta-\gamma}{2}+\frac{1}{q_{2}}-\frac{1}{q_{1}}}}+\int_{0}^{\frac{t}{2}} \frac{L_{\frac{20}{17}}(T) N_{\frac{20}{13}}(T)}{(t-s)^{\frac{27}{20}+\frac{\delta}{2}-\frac{1}{q_{1}}} s^{\frac{3}{20}}} d s+\int_{\frac{t}{2}}^{t} \frac{L_{2}(T) N_{20}(T)}{(t-s)^{\frac{2}{5}+\frac{\delta}{2}-\frac{1}{q_{1}}} s^{\frac{11}{10}}} d s \\
\lesssim \frac{\left\|r^{-\gamma} u_{0}^{\theta}\right\|_{L^{q_{2}}(\Omega)}}{t^{\frac{\delta-\gamma}{2}+\frac{1}{q_{2}}-\frac{1}{q_{1}}}}+\frac{L_{\frac{20}{17}}(T) N_{\frac{20}{13}}(T)+L_{2}(T) N_{20}(T)}{t^{\frac{1}{2}+\frac{\delta}{2}-\frac{1}{q_{1}}}}
\end{gathered}
$$

This proves the estimate (3.28). 
To handle the estimate (3.29), we first consider the case when $\delta \in\left[0, \frac{1}{2}\right]$ and $p \in[1,5]$. In this case, applying Proposition 2.2 to (3.1) and then using the estimate (3.28) gives rise to

$$
\begin{aligned}
& \left\|r^{-\delta} \omega^{\theta}(t)\right\|_{L^{p}(\Omega)} \lesssim \frac{\left\|\omega_{0}^{\theta}\right\|_{L^{1}(\Omega)}}{t^{\frac{\delta}{2}+1-\frac{1}{p}}}+\int_{0}^{\frac{t}{2}}\left(\frac{\|\widetilde{u}(s)\|_{L^{4}(\Omega)}\left\|\omega^{\theta}(s)\right\|_{L^{\frac{4}{3}(\Omega)}}}{(t-s)^{\frac{3}{2}+\frac{\delta}{2}-\frac{1}{p}}}+\frac{\left\|u^{\theta}(s)\right\|_{L^{2}(\Omega)}^{2}}{(t-s)^{2+\frac{\delta}{2}-\frac{1}{p}}}\right) d s \\
& +\int_{\frac{t}{2}}^{t}\left(\frac{\|\widetilde{u}(s)\|_{L^{4 p}(\Omega)}\left\|\omega^{\theta}(s)\right\|_{L^{\frac{4 p}{3}(\Omega)}}}{(t-s)^{\frac{1}{2}+\frac{\delta}{2}}}+\frac{\left\|r^{-\frac{1+\delta}{2}} u^{\theta}(s)\right\|_{L^{2 p}(\Omega)}^{2}}{(t-s)^{\frac{1}{2}}}\right) d s \\
& \lesssim \frac{\left\|\omega_{0}^{\theta}\right\|_{L^{1}(\Omega)}}{t^{\frac{\delta}{2}+1-\frac{1}{p}}}+\int_{0}^{\frac{t}{2}}\left(\frac{L_{\frac{4}{3}}^{2}(T)}{(t-s)^{\frac{3}{2}+\frac{\delta}{2}-\frac{1}{p}} s^{\frac{1}{2}}}+\frac{M_{2}^{2}(T)}{(t-s)^{2+\frac{\delta}{2}-\frac{1}{p}}}\right) d s \\
& +\int_{\frac{t}{2}}^{t}\left(\frac{L_{\frac{4 p}{1+2 p}}(T) L_{\frac{4 p}{3}}(T)}{(t-s)^{\frac{1}{2}+\frac{\delta}{2}} s^{\frac{3}{2}-\frac{1}{p}}}+\frac{L_{\frac{20}{17}, \frac{20}{11}}^{2}(T) N_{\frac{20}{13}, 20}^{2}(T)+\left\|u_{0}^{\theta}\right\|_{L^{2}(\Omega)}^{2}}{(t-s)^{\frac{1}{2}} s^{\frac{3}{2}+\frac{\delta}{2}-\frac{1}{p}}}\right) d s \\
& \lesssim \frac{L_{1, \frac{20}{3}}^{4}(T)+M_{2}^{2}(T)+N_{\frac{20}{13}, 20}^{4}(T)}{t^{\frac{\delta}{2}+1-\frac{1}{p}}},
\end{aligned}
$$

where in the last step we use the fact that $\left\|\omega_{0}^{\theta}\right\|_{L^{1}(\Omega)} \leq L_{1},\left\|u_{0}^{\theta}\right\|_{L^{2}(\Omega)} \leq M_{2}$.

On the other side, when $p \in] 5, \infty]$, we have

$$
\begin{aligned}
\left\|r^{-\delta} \omega^{\theta}(t)\right\|_{L^{p}(\Omega)} \lesssim & \frac{\left\|\omega_{0}^{\theta}\right\|_{L^{1}(\Omega)}}{t^{\frac{\delta}{2}+1-\frac{1}{p}}}+\int_{0}^{\frac{t}{2}}\left(\frac{\|\widetilde{u}(s)\|_{L^{4}(\Omega)}\left\|\omega^{\theta}(s)\right\|_{L^{\frac{4}{3}(\Omega)}}}{(t-s)^{\frac{3}{2}+\frac{\delta}{2}-\frac{1}{p}}}+\frac{\left\|u^{\theta}(s)\right\|_{L^{2}(\Omega)}^{2}}{(t-s)^{2+\frac{\delta}{2}-\frac{1}{p}}}\right) d s \\
& +\int_{\frac{t}{2}}^{t}\left(\frac{\|\widetilde{u}(s)\|_{L^{20}(\Omega)}\left\|\omega^{\theta}(s)\right\|_{L^{\frac{20}{3}}(\Omega)}}{(t-s)^{\frac{7}{10}+\frac{\delta}{2}-\frac{1}{p}}}+\frac{\left\|r^{-\frac{1+\delta}{2}} u^{\theta}(s)\right\|_{L^{2 p}(\Omega)}^{2}}{(t-s)^{\frac{1}{2}}}\right) d s \\
& \lesssim \frac{L_{1, \frac{20}{3}}^{4}(T)+M_{2}^{2}(T)+N_{\frac{20}{13}, 20}^{4}(T)}{t^{\frac{\delta}{2}+1-\frac{1}{p}}}
\end{aligned}
$$

Combining (3.30) with (3.31) leads to the estimate (3.29) for $\delta \in\left[0, \frac{1}{2}\right]$.

For the remaining case when $\delta \in] 1 / 2,1]$, we first get, by a similar derivation of (3.30) and then using (3.28) that for $p \in[1,5]$

$$
\begin{aligned}
\left\|r^{-\delta} \omega^{\theta}(t)\right\|_{L^{p}(\Omega)} \lesssim & \frac{\left\|\omega_{0}^{\theta}\right\|_{L^{1}(\Omega)}}{t^{\frac{\delta}{2}+1-\frac{1}{p}}}+\int_{0}^{\frac{t}{2}}\left(\frac{\|\widetilde{u}(s)\|_{L^{4}(\Omega)}\left\|\omega^{\theta}(s)\right\|_{L^{\frac{4}{3}(\Omega)}}}{(t-s)^{\frac{3}{2}+\frac{\delta}{2}-\frac{1}{p}}}+\frac{\left\|u^{\theta}(s)\right\|_{L^{2}(\Omega)}^{2}}{(t-s)^{2+\frac{\delta}{2}-\frac{1}{p}}}\right) d s \\
& +\int_{\frac{t}{2}}^{t}\left(\frac{\|\widetilde{u}(s)\|_{L^{4 p}(\Omega)}\left\|r^{-\frac{1}{2}} \omega^{\theta}(s)\right\|_{L^{\frac{4 p}{3}}(\Omega)}}{(t-s)^{\frac{1}{4}+\frac{\delta}{2}}}+\frac{\left\|r^{-\frac{1+\delta}{2}} u^{\theta}(s)\right\|_{L^{2 p}(\Omega)}^{2}}{(t-s)^{\frac{1}{2}}}\right) d s \\
& \lesssim \frac{L_{1, \frac{20}{3}}^{5}(T)+M_{2}^{3}(T)+N_{\frac{20}{13}, 20}^{5}(T)}{t^{\frac{\delta}{2}+1-\frac{1}{p}}} .
\end{aligned}
$$


Finally when $p \in] 5, \infty]$, we deduce, by a similar derivation of $(3.31)$, that

$$
\begin{aligned}
\left\|r^{-\delta} \omega^{\theta}(t)\right\|_{L^{p}(\Omega)} \lesssim & \frac{\left\|\omega_{0}^{\theta}\right\|_{L^{1}(\Omega)}}{t^{\frac{\delta}{2}+1-\frac{1}{p}}}+\int_{0}^{\frac{t}{2}}\left(\frac{\|\widetilde{u}(s)\|_{L^{4}(\Omega)}\left\|\omega^{\theta}(s)\right\|_{L^{\frac{4}{3}(\Omega)}}}{(t-s)^{\frac{3}{2}+\frac{\delta}{2}-\frac{1}{p}}}+\frac{\left\|u^{\theta}(s)\right\|_{L^{2}(\Omega)}^{2}}{(t-s)^{2+\frac{\delta}{2}-\frac{1}{p}}}\right) d s \\
& +\int_{\frac{t}{2}}^{t}\left(\frac{\|\widetilde{u}(s)\|_{L^{20}(\Omega)}\left\|r^{-\frac{1}{2}} \omega^{\theta}(s)\right\|_{L^{\frac{20}{3}}(\Omega)}}{(t-s)^{\frac{9}{20}+\frac{\delta}{2}-\frac{1}{p}}}+\frac{\left\|r^{-\frac{1+\delta}{2}} u^{\theta}(s)\right\|_{L^{2 p}(\Omega)}^{2}}{(t-s)^{\frac{1}{2}}}\right) d s \\
& \lesssim \frac{L_{1, \frac{20}{3}}^{5}(T)+M_{2}^{3}(T)+N_{\frac{20}{13}, 20}^{5}(T)}{t^{\frac{\delta}{2}+1-\frac{1}{p}}} .
\end{aligned}
$$

Combining (3.32) with (3.33) implies (3.29) for $\left.\delta \in] \frac{1}{2}, 1\right]$. This completes the proof of the estimate (3.29) and hence the corollary.

\section{Global a priori estimates of (1.4) With nearly CRitical initial data}

The goal of this section is basically to prove that, as long as the initial data belongs to the almost critical spaces, the system has a unique global solution.

Let us introduce another two variables which are of great importance in our work, namely

$$
\left.\eta \stackrel{\text { def }}{=} \frac{\omega^{\theta}}{r}, \quad V^{\varepsilon} \stackrel{\text { def }}{=} \frac{u^{\theta}}{r^{1-\varepsilon}} \quad \text { for any } \varepsilon \in\right] 0,1[\text {. }
$$

And it is not difficult to deduce the equations for $\eta$ and $V^{\varepsilon}$ from (1.4) that

$$
\left\{\begin{array}{l}
\partial_{t} \eta+\left(u^{r} \partial_{r}+u^{z} \partial_{z}\right) \eta-\left(\Delta+\frac{2}{r} \partial_{r}\right) \eta-\frac{2 V \partial_{z} V}{r^{2 \varepsilon}}=0 \\
\partial_{t} V+\left(u^{r} \partial_{r}+u^{z} \partial_{z}\right) V+(2-\varepsilon) \frac{u^{r} V}{r}-\left(\Delta+2(1-\varepsilon) \frac{\partial_{r}}{r}\right) V+\left(2 \varepsilon-\varepsilon^{2}\right) \frac{V}{r^{2}}=0 \\
\left.\eta\right|_{t=0}=\eta_{0}=\frac{\omega_{0}^{\theta}}{r},\left.V\right|_{t=0}=V_{0}=\frac{u_{0}^{\theta}}{r^{1-\varepsilon}},
\end{array}\right.
$$

here and in all that follows, we always denote $V^{\varepsilon}$ as $V$, if there is no ambiguity.

Proposition 4.1. Let $\left(u^{r}, u^{\theta}, u^{z}\right)$ be a smooth enough solution of (1.3) on $[0, T]$. Let $p \in$ ] $\left.1, \frac{21}{20}\right], \varepsilon=\frac{-9 p^{2}+21 p-4}{24 p-2} \in\left[\frac{3251}{9280}, \frac{4}{11}[\right.$, and $q$ be given by

$$
(2-\varepsilon) q=3 p .
$$

We assume that the initial data $\eta_{0} \in L^{p}, V_{0}^{\varepsilon}=\frac{u^{\theta}}{r^{1-\varepsilon}} \in L^{q}, r u_{0}^{\theta} \in L^{\infty} \bigcap L^{\frac{1}{p-1}}$, which satisfy

$$
\left(2 M_{0}\right)^{\frac{3(p+2)}{p(3 p+11)}}\left\|r u_{0}^{\theta}\right\|_{L^{\frac{10(1 p-1)(p+2)(p+3)}{3(p(p+3)(3 p+11)}}}^{\frac{10(12 p-1)}{3 p(p)}}+\left(2 M_{0}\right)^{\frac{p-1}{4 p}}\left\|r u_{0}^{\theta}\right\|_{L^{\frac{1}{3(p-1)^{2}}}}^{\frac{1}{6 p}} \leq c_{0}(p-1),
$$

for some sufficiently small constant $c_{0}$ which does not depend on the choice of $p$, and $M_{0} \stackrel{\text { def }}{=}$ $\left\|V_{0}\right\|_{L^{q}}^{q}+\left\|\eta_{0}\right\|_{L^{p}}^{p}$. Then for any $t \in[0, T]$, we have

$$
\begin{aligned}
\|V(t)\|_{L^{q}}^{q} & +\|\eta(t)\|_{L^{p}}^{p}+\frac{p-1}{2}\left\|\nabla|\eta|^{\frac{p}{2}}\right\|_{L^{2}\left((0, t) \times \mathbb{R}^{3}\right)}^{2} \\
& +\frac{1}{2}\left(\left\|\nabla|V|^{\frac{q}{2}}\right\|_{L^{2}\left((0, t) \times \mathbb{R}^{3}\right)}^{2}+\left\|\frac{|V| \frac{q}{2}}{r}\right\|_{L^{2}\left((0, t) \times \mathbb{R}^{3}\right)}^{2}\right) \leq 2\left(\left\|V_{0}\right\|_{L^{q}}^{q}+\left\|\eta_{0}\right\|_{L^{p}}^{p}\right) .
\end{aligned}
$$

Let us remark that both the index $\frac{10(12 p-1)}{9(p-1)(p+2)(p+3)}$ and $\frac{2}{3(p-1)^{2}}$ are close enough to $\infty$ as long as $p$ approaches 1 , which corresponds to the case with initial data in the critical spaces.

The proof of the above proposition relies on the following lemmas: 
Lemma 4.1. Under the assumptions of Proposition 4.1, for any $t \in] 0, T]$, there holds

$$
\begin{gathered}
\frac{1}{q} \frac{d}{d t}\|V(t)\|_{L^{q}}^{q}+\frac{4(q-1)}{q^{2}}\left\|\nabla|V|^{\frac{q}{2}}\right\|_{L^{2}}^{2}+\left(2 \varepsilon-\varepsilon^{2}\right) \int_{\mathbb{R}^{3}} \frac{|V|^{q}}{r^{2}} d x \\
\lesssim\left\|r u^{\theta}\right\|_{L^{\frac{5}{2-\varepsilon}\left(3-\frac{15}{2-11)}\right.}}^{\frac{5}{(p-1)(p+2)}}\left\|\left.V\right|^{\frac{q}{2}}\right\|_{L^{2}}^{\frac{23-17 p-3 p^{2}}{p(3 p+11)}}\|\eta\|_{L^{p}}^{\frac{3 p^{2}+23 p-11}{2(3 p+11)}} \\
\quad \times\left(\left\|\nabla|\eta|^{\frac{p}{2}}\right\|_{L^{2}}^{2}+\left\|\nabla|V|^{\frac{q}{2}}\right\|_{L^{2}}^{2}+\int_{\mathbb{R}^{3}} \frac{|V|^{q}}{r^{2}} d x\right) .
\end{gathered}
$$

Lemma 4.2. Under the assumptions of Proposition 4.1, for any $t \in] 0, T]$, there holds

$$
\begin{aligned}
\frac{1}{p} \frac{d}{d t} \| & \eta(t)\left\|_{L^{p}}^{p}+\frac{4(p-1)}{p^{2}}\right\| \nabla|\eta|^{\frac{p}{2}} \|_{L^{2}}^{2} \\
& \lesssim\left\|r u^{\theta}\right\|_{L^{\frac{1}{3(p-1)^{2}}}}^{\frac{1}{6 p}}\|\eta\|_{L^{p}}^{\frac{p-1}{4}}\left(\left\|\nabla|\eta|^{\frac{p}{2}}\right\|_{L^{2}}^{2}+\left\|\nabla|V|^{\frac{q}{2}}\right\|_{L^{2}}^{2}+\left\|\frac{|V|^{q}}{r^{2}}\right\|_{L^{1}}\right) .
\end{aligned}
$$

Let us admit the above lemmas and continue our proof of Proposition 4.1.

Proof of Proposition 4.1. By virtue of Lemma 2.1, we get, by summarizing (4.6) for $\varepsilon=$ $\frac{-9 p^{2}+21 p-4}{24 p-2}$ with (4.7), that

$$
\begin{gathered}
\frac{d}{d t}\left(\|V(t)\|_{L^{q}}^{q}+\|\eta(t)\|_{L^{p}}^{p}\right)+\frac{4(p-1)}{p}\left\|\nabla|\eta|^{\frac{p}{2}}\right\|_{L^{2}}^{2} \\
+\frac{4(q-1)}{q}\left\|\nabla|V|^{\frac{q}{2}}\right\|_{L^{2}}^{2}+\frac{3 p\left(-9 p^{2}+21 p-4\right)}{24 p-2}\left\|\frac{|V|^{q}}{r^{2}}\right\|_{L^{1}} \\
\leq C\left(\left\|r u_{0}^{\theta}\right\|_{L^{\frac{10(12 p-1)}{3 p(p+3)(3 p+11)}}}^{\frac{10(12 p-1)}{9(p-1)(p+2)(p+3)}}\left\||V|^{\frac{q}{2}}\right\|_{L^{2}}^{\frac{23-17 p-3 p^{2}}{p(3 p+11)}}\|\eta\|_{L^{p}}^{\frac{3 p^{2}+23 p-11}{2(3 p+11)}}+\left\|r u_{0}^{\theta}\right\|_{L^{\frac{1}{6 p}}}^{\frac{1}{3(p-1)^{2}}}\|\eta\|_{L^{p}}^{\frac{p-1}{4}}\right) \\
\times\left(\left\|\nabla|\eta|^{\frac{p}{2}}\right\|_{L^{2}}^{2}+\left\|\nabla|V|^{\frac{q}{2}}\right\|_{L^{2}}^{2}+\left\|\frac{|V|^{q}}{r^{2}}\right\|_{L^{1}}\right) .
\end{gathered}
$$

Let $M_{0} \stackrel{\text { def }}{=}\left\|V_{0}\right\|_{L^{q}}^{q}+\left\|\eta_{0}\right\|_{L^{p}}^{p}$, and let $T^{\prime}>0$ be determined by

$$
T^{\prime} \stackrel{\text { def }}{=} \sup \left\{t \in[0, T]: \sup _{t^{\prime} \in[0, t]}\left(\|V(t)\|_{L^{q}}^{q}+\|\eta(t)\|_{L^{p}}^{p}\right) \leq 2 M_{0}\right\} .
$$

If $T^{\prime}<T$, then for $t \leq T^{\prime}$, we deduce from the (4.8) that

$$
\begin{array}{r}
\frac{d}{d t}\left(\|V(t)\|_{L^{q}}^{q}+\|\eta(t)\|_{L^{p}}^{p}\right)+(p-1)\left\|\nabla|\eta|^{\frac{p}{2}}\right\|_{L^{2}}^{2}+\left\|\nabla|V|^{\frac{q}{2}}\right\|_{L^{2}}^{2}+\left\|\frac{|V|^{q}}{r^{2}}\right\|_{L^{1}} \\
\leq C\left(\left(2 M_{0}\right)^{\frac{3(p+2)}{p(3 p+11)}}\left\|r u_{0}^{\theta}\right\|^{\frac{10(12 p-1)}{3 p(p+3)(3 p+11)}}+\left(2 M_{0}\right)^{\frac{p-1}{4 p}}\left\|r u_{0}^{\theta}\right\|_{L^{\frac{1}{6 p(12 p-1)}}}^{\frac{1}{3(p-1)^{2}}}\right) \\
\quad \times\left(\left\|\nabla|\eta|^{\frac{p}{2}}\right\|_{L^{2}}^{2}+\left\|\nabla|V|^{\frac{q}{2}}\right\|_{L^{2}}^{2}+\left\|\frac{|V|^{q}}{r^{2}}\right\|_{L^{1}}\right),
\end{array}
$$

which together with (4.4) ensures that for any $t$ in $\left[0, T^{\prime}\right]$,

$$
\frac{d}{d t}\left(\|V(t)\|_{L^{q}}^{q}+\|\eta(t)\|_{L^{p}}^{p}\right)+\frac{p-1}{2}\left\|\nabla|\eta|^{\frac{p}{2}}\right\|_{L^{2}}^{2}+\frac{1}{2}\left\|\nabla|V|^{\frac{q}{2}}\right\|_{L^{2}}^{2}+\frac{1}{2}\left\|\frac{|V|^{q}}{r^{2}}\right\|_{L^{1}} \leq 0 .
$$

This in particular gives rise to

$$
\|V(t)\|_{L^{q}}^{q}+\|\eta(t)\|_{L^{p}}^{p} \leq M_{0} \quad \text { for any } \quad t \leq T^{\prime} .
$$


This contradicts with the definition of $T^{\prime}$ given by (4.9). As a result, it comes out $T^{\prime}=T$, and there holds (4.5) for any $t \in[0, T]$. this completes the proof of the proposition.

Let us now turn to the proof of Lemmas 4.1 and 4.2 .

Proof of Lemma 4.1. For any $\left.p \in] 1, \frac{21}{20}\right]$ and $q$ given by (4.3), we get, by multiplying the second equation of (4.2) by $|V|^{q-2} V$ and then integrating the resulting equality over $\mathbb{R}^{3}$, that

$$
\begin{aligned}
& \frac{1}{q} \frac{d}{d t}\|V(t)\|_{L^{q}}^{q}+\frac{1}{q} \int_{\mathbb{R}^{3}}\left(u^{r} \partial_{r}+u^{z} \partial_{z}\right)|V|^{q} d x+(2-\varepsilon) \int_{\mathbb{R}^{3}} \frac{u^{r}}{r}|V|^{q} d x \\
& \quad-\left.\int_{\mathbb{R}^{3}} \Delta V|| V\right|^{q-2} V d x-\frac{2(1-\varepsilon)}{q} \int_{\mathbb{R}^{3}} \partial_{r}|V|^{q} \frac{1}{r} d x+\left(2 \varepsilon-\varepsilon^{2}\right) \int_{\mathbb{R}^{3}} \frac{|V|^{q}}{r^{2}} d x=0 .
\end{aligned}
$$

Using the fact that $\partial_{r}\left(r u^{r}\right)+\partial_{z}\left(r u^{z}\right)=0$, which implies $\int_{\mathbb{R}^{3}}\left(u^{r} \partial_{r}+u^{z} \partial_{z}\right)|V|^{q} d x=0$, and the homogeneous Dirichlet boundary condition for $u^{r}$ on $r=0$, we deduce

$$
\begin{aligned}
\frac{1}{q} \frac{d}{d t}\|V(t)\|_{L^{q}}^{q}+(2-\varepsilon) & \int_{\mathbb{R}^{3}} \frac{u^{r}}{r}|V|^{q} d x+\left.\frac{2(1-\varepsilon)}{q} \int_{-\infty}^{+\infty}|V|^{q}\right|_{r=0} d z \\
& +\frac{4(q-1)}{q^{2}}\left\|\nabla|V|^{\frac{q}{2}}\right\|_{L^{2}}^{2}+\left(2 \varepsilon-\varepsilon^{2}\right) \int_{\mathbb{R}^{3}} \frac{|V|^{q}}{r^{2}} d x=0,
\end{aligned}
$$

which implies

$$
\frac{1}{q} \frac{d}{d t}\|V(t)\|_{L^{q}}^{q}+\frac{4(q-1)}{q^{2}}\left\|\nabla|V|^{\frac{q}{2}}\right\|_{L^{2}}^{2}+\left(2 \varepsilon-\varepsilon^{2}\right) \int_{\mathbb{R}^{3}} \frac{|V|^{q}}{r^{2}} d x \lesssim \int_{\mathbb{R}^{3}}\left|\frac{u^{r}}{r}\right| \cdot|V|^{q} d x
$$

Let us take $q_{1}, q_{2}$ satisfying $\frac{1}{q_{1}}+\frac{1}{q_{2}}=1$, and temporarily assume that $\left.q_{1} \in\right] \frac{3 p}{3-p}, \infty[$, so that it follows from Lemma 2.1 and Sobolev embedding Theorem that

$$
\begin{aligned}
\int_{\mathbb{R}^{3}}\left|\frac{u^{r}}{r}\right| \cdot|V|^{q} d x & \leq\left\|\frac{u^{r}}{r}\right\|_{L^{q_{1}}}\left\||V|^{q}\right\|_{L^{q_{2}}} \\
& \lesssim\|\eta\|_{L^{p}}^{\frac{p-1}{2}+\frac{3 p}{2 q_{1}}}\|\eta\|_{L^{3 p}}^{\frac{3-p}{2}-\frac{3 p}{2 q_{1}}}\left\||V|^{q}\right\|_{L^{q_{2}}} \\
& \lesssim\|\eta\|_{L^{p}}^{\frac{p-1}{2}+\frac{3 p}{2 q_{1}}}\left\|\nabla|\eta|^{\frac{p}{2}}\right\|_{L^{2}}^{2\left(\frac{3-p}{2 p}-\frac{3}{2 q_{1}}\right)}\left\||V|^{q}\right\|_{L^{q_{2}}}
\end{aligned}
$$

Take $q_{2}=\vartheta+\sigma+\frac{2 \sigma}{3 p}$, with $\vartheta>0$ and $0<\sigma<1$ to be determined later, then we have

$$
\begin{aligned}
\left\||V|^{q}\right\|_{L^{q_{2}}} & =\left(\int_{\mathbb{R}^{3}}|V|^{q \vartheta}\left(\frac{|V|^{q}}{r^{2}}\right)^{\sigma}\left|r^{2-\varepsilon} V\right|^{\frac{2 \sigma}{2-\varepsilon}} d x\right)^{\frac{1}{q_{2}}} \\
& \leq\left\|r u^{\theta}\right\|_{L^{\frac{2 \sigma}{2-\varepsilon} \cdot \frac{p}{p-1}}}^{\frac{2 \sigma}{q_{2}^{(2-\varepsilon)}}}\left\||V|^{\frac{q}{2}}\right\|_{L^{\frac{2 \vartheta}{1-p \sigma}}}^{\frac{2 p \vartheta}{1-p \sigma}}\left(\int_{\mathbb{R}^{3}} \frac{|V|^{q}}{r^{2}} d x\right)^{\frac{\sigma}{q_{2}}} \\
& \leq\left\|r u^{\theta}\right\|_{L^{\frac{2 \sigma}{2-\varepsilon} \cdot \frac{p}{p-1}}}^{\frac{2 \sigma}{q_{2}(2-\varepsilon)}}\left\|\left.V\right|^{\frac{q}{2}}\right\|_{L^{2}}^{\frac{-\vartheta-3 \sigma}{q_{2}}+\frac{3}{p q_{2}}}\left\|\nabla|V|^{\frac{q}{2}}\right\|_{L^{2}}^{\frac{3(\vartheta+\sigma)}{q_{2}}-\frac{3}{p q_{2}}}\left(\int_{\mathbb{R}^{3}} \frac{|V|^{q}}{r^{2}} d x\right)^{\frac{\sigma}{q_{2}}},
\end{aligned}
$$

where in the last step, we use the interpolation inequality provided that

$$
\frac{2 p \vartheta}{1-p \sigma} \in[2,6]
$$


which will be verified later. Then we get, by applying Young's inequality, that

$$
\begin{aligned}
& \int_{\mathbb{R}^{3}}\left|\frac{u^{r}}{r}\right||V|^{q} d x \lesssim\left\|r u^{\theta}\right\|_{L^{\frac{2 \sigma}{2-\varepsilon} \cdot \frac{p}{p-1}}}^{\frac{2 \sigma}{q_{2}(2-\varepsilon)}}\left\||V|^{\frac{q}{2}}\right\|_{L^{2}}^{\frac{-\vartheta-3 \sigma}{q_{2}}+\frac{3}{p q_{2}}}\|\eta\|_{L^{p}}^{\frac{p-1}{2}+\frac{3 p}{2 q_{1}}} \\
& \times\left\|\nabla|\eta|^{\frac{p}{2}}\right\|_{L^{2}}^{2\left(\frac{3-p}{2 p}-\frac{3}{2 q_{1}}\right)}\left\|\nabla|V|^{\frac{q}{2}}\right\|_{L^{2}}^{\frac{3(\vartheta+\sigma)}{q_{2}}-\frac{3}{p q_{2}}}\left(\int_{\mathbb{R}^{3}} \frac{|V|^{q}}{r^{2}} d x\right)^{\frac{\sigma}{q_{2}}} \\
& \lesssim\left\|r u^{\theta}\right\|_{L^{\frac{2 \sigma}{2-\varepsilon} \cdot \frac{p}{p-1}}}^{\frac{2 \sigma}{q_{2}(2-\varepsilon)}}\left\||V|^{\frac{q}{2}}\right\|_{L^{2}}^{\frac{-\vartheta-3 \sigma}{q_{2}}+\frac{3}{p q_{2}}}\|\eta\|_{L^{p}}^{\frac{p-1}{2}+\frac{3 p}{2 q_{1}}} \\
& \times\left(\left\|\nabla|\eta|^{\frac{p}{2}}\right\|_{L^{2}}^{2}+\left\|\nabla|V|^{\frac{q}{2}}\right\|_{L^{2}}^{2}+\int_{\mathbb{R}^{3}} \frac{|V|^{q}}{r^{2}} d x\right),
\end{aligned}
$$

provided that

$$
\frac{3-p}{2 p}-\frac{3}{2 q_{1}}+\frac{3(\vartheta+\sigma)}{2 q_{2}}-\frac{3}{2 p q_{2}}+\frac{\sigma}{q_{2}}=1,
$$

and this can be satisfied by choosing

$$
\sigma=\frac{5}{2(p+2)}, \vartheta=1-\frac{5}{6 p}, \text { and } q_{2}=\vartheta+\sigma+\frac{2 \sigma}{3 p}=\frac{3 p+11}{3(p+2)} .
$$

It is easy to verify that for any $\left.p \in] 1, \frac{21}{20}\right]$, the corresponding $\frac{2 p \vartheta}{1-p \sigma}=\frac{2(p+2)(6 p-5)}{3(4-3 p)}$ is exactly in $[2,6]$, and $q_{2}$ is exactly in $] 1, \frac{3 p}{4 p-3}\left[\right.$, thus the conjugate number $q_{1}=\left(1-\frac{1}{q_{2}}\right)^{-1}>\frac{3 p}{3-p}$. Thus all the above calculations go through.

With the indexes given by (4.16), by inserting the Estimate (4.15) into (4.12), we achieve (4.6). This completes the proof of Lemma 4.1.

Proof of Lemma 4.2. Analogue to the proof of Lemma 4.1, we get, by first multiplying the $\eta$ equation of (4.2) by $|\eta|^{p-2} \eta$ and then integrating the resulting equality over $\mathbb{R}^{3}$, that

$$
\begin{aligned}
\frac{1}{p} \frac{d}{d t}\|\eta(t)\|_{L^{p}}^{p}+\frac{1}{p} \int_{\mathbb{R}^{3}} & \left(u^{r} \partial_{r}+u^{z} \partial_{z}\right)|\eta|^{p} d x \\
& \quad-\left.\int_{\mathbb{R}^{3}} \Delta \eta|| \eta\right|^{p-2} \eta d x-\frac{2}{p} \int_{\mathbb{R}^{3}} \frac{\partial_{r}|\eta|^{p}}{r} d x=\int_{\mathbb{R}^{3}} \frac{2 V \partial_{z} V}{r^{2 \varepsilon}}|\eta|^{p-2} \eta d x .
\end{aligned}
$$

Once again due to $\partial_{r}\left(r u^{r}\right)+\partial_{z}\left(r u^{z}\right)=0$ and $\left.u^{r}\right|_{r=0}=0$, we get

$$
\frac{1}{p} \frac{d}{d t}\|\eta(t)\|_{L^{p}}^{p}+\frac{4(p-1)}{p^{2}}\left\|\nabla|\eta|^{\frac{p}{2}}\right\|_{L_{x}^{2}}^{2}+\left.\frac{2}{p} \int_{-\infty}^{+\infty}|\eta|^{p}\right|_{r=0} d z=\int_{\mathbb{R}^{3}} \frac{2 V \partial_{z} V}{r^{2 \varepsilon}}|\eta|^{p-2} \eta d x .
$$

It is easy to observe that

$$
\begin{aligned}
\left.\left|\int_{\mathbb{R}^{3}} \frac{2 V \partial_{z} V}{r^{2 \varepsilon}}\right| \eta\right|^{p-2} \eta d x \mid & \leq\left.\frac{2}{q} \int_{\mathbb{R}^{3}}|\eta|^{p-1}\left|\partial_{z}\right| V\right|^{\frac{q}{2}} \mid \frac{|V|^{2-\frac{q}{2}}}{r^{2 \varepsilon}} d x \\
& \lesssim\left\||\eta|^{p-1}\right\|_{L^{\frac{2 p}{p-1}}}\left\|\partial_{z}|V|^{\frac{q}{2}}\right\|_{L^{2}}\left\|\frac{|V|^{2-\frac{q}{2}}}{r^{2 \varepsilon}}\right\|_{L^{2 p}} .
\end{aligned}
$$

It follows from Sobolev embedding Theorem that

$$
\begin{aligned}
\left\||\eta|^{p-1}\right\|_{L^{\frac{2 p}{p-1}}}=\left\||\eta|^{\frac{p}{2}}\right\|_{L^{4}}^{\frac{2(p-1)}{p}} & \lesssim\left\||\eta|^{\frac{p}{2}}\right\|_{L^{2}}^{\frac{p-1}{2 p}}\left\|\nabla|\eta|^{\frac{p}{2}}\right\|_{L^{2}}^{\frac{3(p-1)}{2 p}} \\
& =\|\eta\|_{L^{p}}^{\frac{p-1}{4}}\left\|\nabla|\eta|^{\frac{p}{2}}\right\|_{L^{2}}^{\frac{3(p-1)}{2 p}} .
\end{aligned}
$$


As a result, we obtain

$$
\left.\left|\int_{\mathbb{R}^{3}} \frac{2 V \partial_{z} V}{r^{2 \varepsilon}}\right| \eta\right|^{p-2} \eta d x\left|\lesssim\|\eta\|_{L^{p}}^{\frac{p-1}{4}}\left\|\nabla|\eta|^{\frac{p}{2}}\right\|_{L^{2}}^{\frac{3(p-1)}{2^{2 p}}}\left\|\partial_{z}|V|^{\frac{q}{2}}\right\|_{L^{2}}\left\|\frac{|V|^{2-\frac{q}{2}}}{r^{2 \varepsilon}}\right\|_{L^{2 p}} .\right.
$$

To handle the term $\left\|\frac{|V|^{2-\frac{q}{2}}}{r^{2 \varepsilon}}\right\|_{L^{2 p}}$, we split $\frac{|V|^{2-\frac{q}{2}}}{r^{2 \varepsilon}}$ as

$$
\frac{|V|^{2-\frac{q}{2}}}{r^{2 \varepsilon}}=\left|\frac{|V|^{q}}{r^{2}}\right|^{\alpha}\left|r^{2-\varepsilon} V\right|^{\frac{1}{6 p}}|V|^{\frac{q}{2} \cdot \beta}
$$

with $\alpha, \beta$ being determined by

$$
\alpha=\frac{1}{6 p}\left(1-\frac{\varepsilon}{2}\right)+\varepsilon \quad \text { and } \quad \beta=\frac{2}{q}\left(2-\frac{q}{2}-q \alpha-\frac{1}{6 p}\right)=\frac{1}{3 p}\left(7-\frac{2}{3 p}\right)\left(1-\frac{\varepsilon}{2}\right)-1-2 \varepsilon .
$$

It follows from Sobolev embedding Theorem that

$$
\begin{aligned}
\left\||V|^{\frac{q}{2} \cdot \beta}\right\|_{L^{r}} & \lesssim\left\|\nabla|V|^{\frac{q}{2}}\right\|_{L^{2}}^{\frac{3-p}{2 p}-2 \alpha}\left\||V|^{\frac{q}{2}}\right\|_{L^{2}}^{2 \alpha+\beta-\frac{3-p}{2 p}} \text { with } \\
\frac{1}{r} & =\frac{\beta}{2}+\frac{2 \alpha}{3}-\frac{3-p}{6 p}=\frac{1}{18 p}\left(14-\frac{23}{2} \varepsilon-\frac{2-\varepsilon}{p}\right)-\frac{1}{3}(1+\varepsilon) .
\end{aligned}
$$

It is easy to verify that $\alpha+\frac{1}{r}=\frac{1}{2 p}-\frac{(p-1)^{2}}{4 p}$ provided selecting

$$
\varepsilon=\frac{-9 p^{2}+21 p-4}{24 p-2}
$$

which belongs to $\left[\frac{3251}{9280}, \frac{4}{11}[\right.$ whenever $\left.p \in] 1, \frac{21}{20}\right]$.

Moreover, under such choice of indexes, the term $\left\||V|^{\frac{q}{2}}\right\|_{L^{2}}^{2 \alpha+\beta-\frac{3-p}{2 p}}$ in (4.19) disappears. Then we get, by applying Hölder's inequality, that

$$
\begin{aligned}
\left\|\frac{|V|^{2-\frac{q}{2}}}{r^{2 \varepsilon}}\right\|_{L^{2 p}} & \leq\left\|\left(\frac{|V|^{q}}{r^{2}}\right)^{\alpha}\right\|_{L^{\frac{1}{\alpha}}}\left\||V|^{\frac{q}{2} \cdot \beta}\right\|_{L^{r}}\left\|\left|r^{2-\varepsilon} V\right|^{\frac{1}{6 p}}\right\|_{L^{\frac{4 p}{(p-1)^{2}}}} \\
& \leq\left\|\frac{|V|^{q}}{r^{2}}\right\|_{L^{1}}^{\alpha}\left\|\nabla|V|^{\frac{q}{2}}\right\|_{L^{2}}^{\frac{3-p}{2 p}-2 \alpha}\left\|r u^{\theta}\right\|_{L^{\frac{1}{3(p-1)^{2}}}}^{\frac{1}{6 p}} .
\end{aligned}
$$

Inserting the above inequality into (4.18) gives rise to

$$
\left.\left|\int_{\mathbb{R}^{3}} \frac{2 V \partial_{z} V}{r^{2 \varepsilon}}\right| \eta\right|^{p-2} \eta d x\left|\lesssim\|\eta\|_{L^{p}}^{\frac{p-1}{4}}\left\|\nabla|\eta|^{\frac{p}{2}}\right\|_{L^{2}}^{\frac{3(p-1)}{2 p}}\left\|\nabla|V|^{\frac{q}{2}}\right\|_{L^{2}}^{\frac{3-p}{2 p}+1-2 \alpha}\left\|\frac{|V|^{q}}{r^{2}}\right\|_{L^{1}}^{\alpha}\left\|r u^{\theta}\right\|_{L^{\frac{1}{3(p-1)^{2}}}}^{\frac{1}{6 p}} .\right.
$$

Note that $\frac{3(p-1)}{2 p}+\left(\frac{3-p}{2 p}+1-2 \alpha\right)+2 \alpha=2$, by substituting the above inequality into (4.17) and using Young's inequality, we achieve (4.7). This completes the proof of Lemma 4.2.

\section{Global Well-Posedness With CRItical initial Data}

In what follows, we shall always denote $U \stackrel{\text { def }}{=} \frac{u^{\theta}}{r}$ and $W \stackrel{\text { def }}{=} r^{-\frac{7}{11}} u^{\theta}$. 
Lemma 5.1. Under the assumption of Theorem 1.2, for any $p \in\left[1, \frac{21}{20}\right], \varepsilon=\frac{-9 p^{2}+21 p-4}{24 p-2}$, and $q=\frac{3 p}{2-\varepsilon}$, the local solutions constructed in Theorem 1.1 satisfy

$$
\begin{aligned}
\|\eta(t)\|_{L^{p}} & \left.\left.\leq \frac{C}{t^{\frac{3(p-1)}{2 p}}} \cdot\left(L_{1, \frac{20}{3}}^{5}(T)+M_{2}^{3}(T)+N_{\frac{20}{13}, 20}^{5}(T)\right) \quad \forall t \in\right] 0, T\right], \\
\|U(t)\|_{L^{\frac{3}{2}}} & \left.\left.\leq C\left(\left\|U_{0}\right\|_{L^{\frac{3}{2}}}+L_{\frac{20}{17}, 2}(T) N_{\frac{20}{13}, 20}(T)\right) \quad \forall t \in\right] 0, T\right], \\
\left\|V^{\varepsilon}(t)\right\|_{L^{q}} & \left.\left.\leq \frac{C}{t^{\frac{3(p-1)}{2 q}}}\left(\left\|U_{0}\right\|_{L^{\frac{3}{2}}}^{\frac{9}{11}}\left\|r u_{0}^{\theta}\right\|_{L^{\infty}}^{\frac{2}{11}}+L_{\frac{20}{17}, 2}^{2}(T)+N_{\frac{20}{13}, 20}^{2}(T)\right) \quad \forall t \in\right] 0, T\right] .
\end{aligned}
$$

Proof. Taking $\delta=1-\frac{1}{p}$ in (3.29) yields (5.1). Likewise, taking $\delta=\gamma=\frac{1}{3}$ and $q_{1}=q_{2}=\frac{3}{2}$ in (3.28) leads to (5.2).

On the other hand, for any $p \in[1,21 / 20]$, due to the choice of $\varepsilon$ and $q$, we have

$$
1-\varepsilon-\frac{1}{q}=\frac{9 p^{2}-7}{24 p-2} \geq \frac{1}{11}, \quad q=\frac{24 p-2}{3 p+9} \geq \frac{11}{6} .
$$

Noting that $\left\|V^{\varepsilon}(t)\right\|_{L^{q}}=\left\|\frac{u^{\theta}}{r^{1-\varepsilon-\frac{1}{q}}}\right\|_{L^{q}(\Omega)}$ and $\left\|r^{-\frac{7}{11}} u_{0}^{\theta}\right\|_{L^{\frac{11}{6}}}=\left\|r^{-\frac{1}{11}} u_{0}^{\theta}\right\|_{L^{\frac{11}{6}(\Omega)}}$, then applying (3.28) with $q_{1}=q, q_{2}=\frac{11}{6}, \delta=1-\varepsilon-\frac{1}{q}$ and $\gamma=\frac{1}{11}$ gives

$$
\begin{aligned}
\left\|V^{\varepsilon}(t)\right\|_{L^{q}} & \leq \frac{C}{t^{\frac{3(p-1)}{2 q}}} \cdot\left(\left\|r^{-\frac{7}{11}} u_{0}^{\theta}\right\|_{L^{\frac{11}{6}}}+L_{\frac{20}{17}, 2}(T) N_{\frac{20}{13}, 20}(T)\right) \\
& \left.\left.\leq \frac{C}{t^{\frac{3(p-1)}{2 q}}} \cdot\left(\left\|U_{0}\right\|_{L^{\frac{3}{2}}}^{\frac{9}{11}}\left\|r u_{0}^{\theta}\right\|_{L^{\infty}}^{\frac{2}{11}}+L_{\frac{20}{17}, 2}^{2}(T)+N_{\frac{20}{13}, 20}^{2}(T)\right), \quad \forall t \in\right] 0, T\right],
\end{aligned}
$$

where we have used Hölder's inequality in the last step.

Proof of Theorem 1.2. Due to $U_{0}=\frac{u_{0}^{\theta}}{r} \in L^{\frac{3}{2}}, r u_{0}^{\theta} \in L^{\infty}$, and

$$
\left.\left\|r^{\kappa} u_{0}^{\theta}\right\|_{L^{\frac{3}{1-\kappa}}} \leq\left\|U_{0}\right\|_{L^{\frac{3}{2}}}^{\frac{1-\kappa}{2}}\left\|r u_{0}^{\theta}\right\|_{L^{\infty}}^{\frac{1+\kappa}{2}}, \forall \kappa \in\right]-1,1[
$$

we deduce that both $\left\|u_{0}^{\theta}\right\|_{L^{2}(\Omega)}=\left\|r^{-\frac{1}{2}} u_{0}^{\theta}\right\|_{L^{2}}$ and $\left\|r^{-\frac{3}{10}} u_{0}^{\theta}\right\|_{L^{\frac{20}{13}(\Omega)}}=\left\|r^{-\frac{19}{20}} u_{0}^{\theta}\right\|_{L^{\frac{20}{13}}}$ are sufficiently small as long as $\left\|r u_{0}^{\theta}\right\|_{L^{\infty}}$ is small enough. Then by Theorem 1.1, the equation (1.4) has a unique mild solution

$$
\begin{gathered}
\left.\left.\left.\left.\omega^{\theta} \in C\left([0, T] ; L^{1}(\Omega)\right) \bigcap C(] 0, T\right] ; L^{\infty}(\Omega)\right), u^{\theta} \in C\left([0, T] ; L^{2}(\Omega)\right) \bigcap C(] 0, T\right] ; L^{\infty}(\Omega)\right) \\
\text { with } \left.\left.r^{-\frac{3}{10}} u^{\theta} \in C\left([0, T] ; L^{\frac{20}{13}}(\Omega)\right) \bigcap C(] 0, T\right] ; L^{\infty}(\Omega)\right),
\end{gathered}
$$

and the lifespan $T>0$ depends only on $\omega_{0}^{\theta}$. We denote $t_{0} \stackrel{\text { def }}{=} \frac{T}{2}$. In the following, we will always abbreviate $L_{p}(T)$ as $L_{p}$, similar abbreviations for the remaining ones in (1.7), (3.27).

If $r u^{\theta}\left(t_{0}\right) \in L^{A} \cap L^{\infty}$ and if there exists some $\left.p_{0} \in\right] 1, \min \left(1+\frac{1}{10 A}, \frac{21}{20}\right)[$, it follows from Proposition 4.1 that for $\varepsilon_{0} \stackrel{\text { def }}{=} \frac{-9 p_{0}^{2}+21 p_{0}-4}{24 p_{0}-2}$ and $q_{0} \stackrel{\text { def }}{=} \frac{2\left(12 p_{0}-1\right)}{3(p+3)}$, if there holds

$$
\left(2 M_{1}\right)^{\frac{3\left(p_{0}+2\right)}{p_{0}\left(3 p_{0}+11\right)}}\left\|r u^{\theta}\left(t_{0}\right)\right\|_{L^{\alpha\left(p_{0}\right)}}^{\frac{10\left(12 p_{0}-1\right)}{3 p_{0}\left(p_{0}+3\right)\left(3 p_{0}+11\right)}}+\left(2 M_{1}\right)^{\frac{p_{0}-1}{4 p_{0}}}\left\|r u^{\theta}\left(t_{0}\right)\right\|_{L^{\frac{1}{3\left(p_{0}-1\right)^{2}}}}^{\frac{1}{6 p_{0}}} \leq c_{0} \cdot\left(p_{0}-1\right),
$$

where

$$
M_{1} \stackrel{\text { def }}{=}\left\|V^{\varepsilon_{0}}\left(t_{0}\right)\right\|_{L^{q_{0}}}^{q_{0}}+\left\|\eta\left(t_{0}\right)\right\|_{L^{p_{0}}}^{p_{0}} \quad \text { and } \quad \alpha\left(p_{0}\right) \stackrel{\text { def }}{=} \frac{10\left(12 p_{0}-1\right)}{9\left(p_{0}-1\right)\left(p_{0}+2\right)\left(p_{0}+3\right)} .
$$


Then the system (1.4) has a global solution. Moreover, in view of (4.5) and Lemma 5.1, for all $t \in\left[t_{0}, \infty\right)$, there holds

$$
\|\eta(t)\|_{L^{p_{0}}}^{p_{0}}+\frac{p_{0}-1}{2}\left\|\nabla|\eta|^{\frac{p_{0}}{2}}\right\|_{L^{2}\left(\left[t_{0}, t\right) \times \mathbb{R}^{3}\right)}^{2} \leq \frac{C}{t_{0}^{\frac{3\left(p_{0}-1\right)}{2}}}\left(\left\|r u_{0}^{\theta}\right\|_{L^{\infty}}^{q_{0}}+L_{1, \frac{20}{3}}^{5 p_{0}}+M_{2}^{3 p_{0}}+N_{\frac{20}{13}, 20}^{5 p_{0}}\right) .
$$

By the choice of $p_{0}, \alpha\left(p_{0}\right)>10 A$. Then we deduce from Lemma 2.1 and Lemma 5.1 that the smallness condition (5.4) holds provided that

$$
\begin{aligned}
& \left\|r u_{0}^{\theta}\right\|_{L^{\infty}} \leq \frac{c_{0}}{L_{1, \frac{20}{3}}^{6}+M_{2}^{4}+N_{\frac{20}{13}, 20}^{6}} \min \left\{\left(\left(p_{0}-1\right)\left(t_{0}^{\frac{3}{2}}\left\|r u_{0}^{\theta}\right\|_{L^{A}}^{-A}\right)^{\frac{\left(p_{0}-1\right)^{2}}{4 p_{0}}}\right)^{\frac{12 p_{0}}{2-3 A\left(p_{0}-1\right)^{2}}}\right. \\
& \left.\left(\left(p_{0}-1\right)\left(t_{0}^{\frac{3}{2}}\left\|r u_{0}^{\theta}\right\|_{L^{A}}^{-A}\right)^{\frac{3\left(p_{0}-1\right)\left(p_{0}+2\right)}{p_{0}\left(3 p_{0}+11\right)}}\right)^{\frac{3 p_{0}\left(p_{0}+3\right)\left(3 p_{0}+11\right)}{10\left(12 p_{0}-1\right)-9 A\left(p_{0}-1\right)\left(p_{0}+2\right)\left(p_{0}+3\right)}}\right\} .
\end{aligned}
$$

In the following, we consider estimates in critical spaces. By a similar derivation as Lemma 4.1 , that for $q_{1,1}, q_{2,1}$ satisfying $\frac{1}{q_{1,1}}+\frac{1}{q_{2,1}}=1$ and $q_{1,1}>\frac{3 p_{0}}{3-p_{0}}$, there holds

$$
\begin{aligned}
\frac{6}{11} \frac{d}{d t}\|W(t)\|_{L^{\frac{11}{6}}}^{\frac{11}{6}}+\frac{120}{121}\left\|\nabla|W|^{\frac{11}{12}}\right\|_{L^{2}}^{2}+\frac{72}{121} \int_{\mathbb{R}^{3}} \frac{|W|^{\frac{11}{6}}}{r^{2}} d x \lesssim \int_{\mathbb{R}^{3}}\left|\frac{u^{r}}{r}\right| \cdot|W|^{\frac{11}{6}} d x \\
\lesssim\|\eta\|_{L^{p_{0}}}^{\frac{p_{0}-1}{2}+\frac{3 p_{0}}{2 q_{1,1}}}\left\|\nabla|\eta|^{\frac{p_{0}}{2}}\right\|_{L^{2}}^{2\left(\frac{3-p_{0}}{2 p_{0}}-\frac{3}{2 q_{1,1}}\right)}\left\||W|^{\frac{11}{6}}\right\|_{L^{q_{2,1}}}
\end{aligned}
$$

Take $q_{2,1}=\vartheta_{1}+\sigma_{1}+\frac{2 \sigma_{1}}{3}$, with $\vartheta_{1}>0,0<\sigma_{1}<1$ to be determined later, then we have

$$
\begin{aligned}
& \left\||W|^{\frac{11}{6}}\right\|_{L^{q_{2,1}}}=\left(\int_{\mathbb{R}^{3}}|W|^{\frac{11}{6} \vartheta_{1}}\left(\frac{|W|^{\frac{11}{6}}}{r^{2}}\right)^{\sigma_{1}}\left|r^{\frac{18}{11}} W\right|^{\frac{11 \sigma_{1}}{9}} d x\right)^{\frac{1}{q_{2,1}}} \\
& \lesssim\left\|r u^{\theta}\right\|_{L^{\frac{112\left(p_{0} p_{0}\right)}{9 q_{2}-1}}}^{\frac{11 \sigma_{1}}{9 q_{2}}}\left\|\left.W\right|^{\frac{11}{12}}\right\|_{L^{\frac{2 \vartheta_{0}}{4-p_{0}-3 p_{0} \sigma_{1}}}}^{\frac{2 \vartheta_{1}}{q_{2,1}}}\left(\int_{\mathbb{R}^{3}} \frac{|W|^{\frac{11}{6}}}{r^{2}} d x\right)^{\frac{\sigma_{1}}{q_{2,1}}}, \\
& \lesssim\left\|r u^{\theta}\right\|_{L^{12\left(p_{0}-1\right)}}^{\frac{11 \sigma_{1}}{9 q_{2}, 1}}\left\||W|^{\frac{11}{12}}\right\|_{L^{2}}^{\frac{-\vartheta_{1}-3 \sigma_{1}}{q_{2,1}}+\frac{4-p_{0}}{p_{0} q_{2,1}}}\left\|\nabla|W|^{\frac{11}{12}}\right\|_{L^{2}}^{\frac{3\left(\vartheta_{1}+\sigma_{1}\right)}{q_{2,1}}-\frac{4-p_{0}}{p_{0} q_{2,1}}}\left(\int_{\mathbb{R}^{3}} \frac{|W|^{\frac{11}{6}}}{r^{2}} d x\right)^{\frac{\sigma_{1}}{q_{2,1}}},
\end{aligned}
$$

where in the last step, we used Galiardo-Nirenberg inequality provided that

$$
\frac{6 p_{0} \vartheta_{1}}{4-p_{0}-3 p_{0} \sigma_{1}} \in[2,6]
$$

which will be verified later. Then we get, by applying Young's inequality, that

$$
\begin{aligned}
\int_{\mathbb{R}^{3}}\left|\frac{u^{r}}{r}\right||W|^{\frac{11}{6}} d x \lesssim & \left\|r u^{\theta}\right\|_{L^{\frac{111 \sigma_{1}}{9 q_{2}, 1}}}^{\frac{11 \sigma_{1} p_{0}}{12\left(p_{0}-1\right)}}\left\|\left.W\right|^{\frac{11}{12}}\right\|_{L^{2}}^{\frac{-\vartheta_{1}-3 \sigma_{1}}{q_{2,1}}+\frac{4-p_{0}}{p_{0} q_{2,1}}}\|\eta\|_{L^{p_{0}}}^{\frac{p_{0}-1}{2}+\frac{3 p_{0}}{2 q_{1,1}}} \\
& \times\left(\left\|\nabla|\eta|^{\frac{p_{0}}{2}}\right\|_{L^{2}}^{2}+\left\|\nabla|W|^{\frac{11}{12}}\right\|_{L^{2}}^{2}+\int_{\mathbb{R}^{3}} \frac{|W|^{\frac{11}{6}}}{r^{2}} d x\right),
\end{aligned}
$$

provided that

$$
\frac{3-p_{0}}{2 p_{0}}-\frac{3}{2 q_{1,1}}+\frac{3\left(\vartheta_{1}+\sigma_{1}\right)+1}{2 q_{2,1}}-\frac{2}{p_{0} q_{2,1}}+\frac{\sigma_{1}}{q_{2,1}}=1,
$$

which in particular holds by taking

$$
\sigma_{1}=\frac{2}{5}, \vartheta_{1}=\frac{2}{3} \text {, and } q_{2,1}=\vartheta_{1}+\sigma_{1}+\frac{2 \sigma_{1}}{3}=\frac{4}{3} .
$$

So that (5.8) holds and $q_{1,1}=4>\frac{3 p_{0}}{3-p_{0}}$. Hence all the above calculations go through. 
By inserting the Estimate (5.9) into (5.7), with the indices given by (5.10), we obtain

$$
\begin{aligned}
\frac{6}{11} \frac{d}{d t}\|W(t)\|_{L^{\frac{11}{6}}}^{\frac{11}{6}} & +\frac{120}{121}\left\|\nabla|W|^{\frac{11}{12}}\right\|_{L^{2}}^{2}+\frac{72}{121} \int_{\mathbb{R}^{3}} \frac{|W|^{\frac{11}{6}}}{r^{2}} d x \\
\lesssim & \left\|r u^{\theta}\right\|^{\frac{11}{30}} \frac{11 p_{0}}{L^{30\left(p_{0}-1\right)}}\left\||W|^{\frac{11}{12}}\right\|_{L^{2}}^{\frac{3}{p_{0}}-\frac{43}{20}}\|\eta\|_{L^{\frac{p_{0}}{8}}}^{\frac{7 p_{0}}{2}} \\
& \times\left(\left\|\nabla|\eta|^{\frac{p_{0}}{2}}\right\|_{L^{2}}^{2}+\left\|\nabla|W|^{\frac{11}{12}}\right\|_{L^{2}}^{2}+\int_{\mathbb{R}^{3}} \frac{|W|^{\frac{11}{6}}}{r^{2}} d x\right) .
\end{aligned}
$$

Next, applying $L^{1}$ energy estimate for the $\eta$ equation in (4.2) yields

$$
\frac{d}{d t}\|\eta(t)\|_{L^{1}}+\int_{\mathbb{R}^{3}}(-\Delta \eta) \cdot \operatorname{sgn} \eta \mathrm{dx}+2 \int_{-\infty}^{+\infty}|\eta|_{\mathrm{r}=0} \mathrm{dz} \lesssim\left\|\partial_{\mathrm{z}}|\mathrm{W}|^{\frac{11}{12}}\right\|_{\mathrm{L}^{2}}\left\|\frac{|\mathrm{W}|^{\frac{13}{12}}}{\mathrm{r}^{\frac{8}{11}}}\right\|_{\mathrm{L}^{2}} .
$$

Noting that $\frac{|W| \frac{13}{12}}{r \frac{8}{11}}=\left|\frac{|W|^{\frac{11}{6}}}{r^{2}}\right|^{\frac{1}{2}} \cdot\left|r^{\frac{18}{11}} W\right|^{\frac{1}{6}}$, so we have

$$
\left\|\frac{|W|^{\frac{13}{12}}}{r^{\frac{8}{11}}}\right\|_{L^{2}} \leq\left\|r u^{\theta}\right\|_{L^{\infty}}^{\frac{1}{6}} \cdot\left\|\frac{|W|^{\frac{11}{6}}}{r^{2}}\right\|_{L^{1}}^{\frac{1}{2}} .
$$

Substituting (5.13) into (5.12), and using the fact that $\int_{\mathbb{R}^{3}}(-\Delta \eta) \cdot \operatorname{sgn} \eta \mathrm{dx} \leq 0$, we achieve

$$
\begin{aligned}
\frac{d}{d t}\|\eta(t)\|_{L^{1}} & \lesssim\left\|r u^{\theta}\right\|_{L^{\infty}}^{\frac{1}{6}}\left\|\partial_{z}|W|^{\frac{11}{12}}\right\|_{L^{2}}\left\|\frac{|W|^{\frac{11}{6}}}{r^{2}}\right\|_{L^{1}}^{\frac{1}{2}} \\
& \lesssim\left\|r u^{\theta}\right\|_{L^{\infty}}^{\frac{1}{6}}\left(\left\|\partial_{z}|W|^{\frac{11}{12}}\right\|_{L^{2}}^{2}+\left\|\frac{|W|^{\frac{11}{6}}}{r^{2}}\right\|_{L^{1}}\right) .
\end{aligned}
$$

Meanwhile, by taking $p=p_{0}$ in (4.8), we have

$$
\begin{aligned}
& \frac{d}{d t}\left(\left\|V^{\varepsilon_{0}}(t)\right\|_{L^{q_{0}}}^{q_{0}}+\|\eta(t)\|_{L^{p_{0}}}^{p_{0}}\right)+\frac{4\left(p_{0}-1\right)}{p_{0}}\left\|\nabla|\eta|^{\frac{p_{0}}{2}}\right\|_{L^{2}}^{2} \\
& \quad+\frac{4\left(q_{0}-1\right)}{q_{0}}\left\|\nabla\left|V^{\varepsilon_{0}}\right|^{\frac{q_{0}}{2}}\right\|_{L^{2}}^{2}+\frac{3 p_{0}\left(-9 p_{0}^{2}+21 p_{0}-4\right)}{24 p_{0}-2}\left\|\frac{\left|V^{\varepsilon_{0}}\right|^{q_{0}}}{r^{2}}\right\|_{L^{1}} \\
& \lesssim\left(\left\|r u_{0}^{\theta}\right\|_{L^{\alpha\left(p_{0}\right)}}^{\frac{10\left(12 p_{0}-1\right)}{\left.3 p_{0}+3 p_{0}+11\right)}}\left\|V^{\varepsilon_{0}}\right\|_{L^{2}}^{q_{0} \cdot \frac{23-17 p_{0}-3 p_{0}^{2}}{2 p_{0}\left(3 p_{0}+11\right)}}\|\eta\|_{L_{0}^{p}}^{\frac{3 p_{0}^{2}+23 p_{0}-11}{2\left(3 p_{0}+11\right)}}+\left\|r u_{0}^{\theta}\right\|_{L^{\frac{1}{6\left(p_{0}\right.}}}^{\frac{1}{3\left(p_{0}-1\right)^{2}}}\|\eta\|_{L^{p_{0}}}^{\frac{p_{0}-1}{4}}\right) \\
& \quad \times\left(\left\|\nabla|\eta|^{\frac{p_{0}}{2}}\right\|_{L^{2}}^{2}+\left\|\nabla\left|V^{\varepsilon_{0}}\right|^{\frac{q_{0}}{2}}\right\|_{L^{2}}^{2}+\left\|\frac{\left|V^{\varepsilon_{0}}\right| q_{0}}{r^{2}}\right\|_{L^{1}}\right) .
\end{aligned}
$$


Summarizing the estimates (5.11), (5.14) and (5.15) gives rise to

$$
\begin{aligned}
& \frac{d}{d t}\left(\|\eta(t)\|_{L^{1}}+\|W(t)\|_{L^{\frac{11}{6}}}^{\frac{11}{6}}+\left\|V^{\varepsilon_{0}}(t)\right\|_{L^{q_{0}}}^{q_{0}}+\|\eta(t)\|_{L^{p_{0}}}^{p_{0}}\right)+\left(p_{0}-1\right)\left\|\nabla|\eta|^{\frac{p_{0}}{2}}\right\|_{L^{2}}^{2} \\
& +\left\|\nabla\left|V^{\varepsilon_{0}}\right|^{\frac{q_{0}}{2}}\right\|_{L^{2}}^{2}+\left\|\frac{\left|V^{\varepsilon_{0}}\right|^{q_{0}}}{r^{2}}\right\|_{L^{1}}+\left\|\nabla|W|^{\frac{11}{12}}\right\|_{L^{2}}^{2}+\int_{\mathbb{R}^{3}} \frac{|W|^{\frac{11}{6}}}{r^{2}} d x \\
& \lesssim\left(\left\|r u_{0}^{\theta}\right\|_{L^{\alpha\left(p_{0}\right)}}^{\frac{10\left(12 p_{0}-1\right)}{3 p_{0}\left(p_{0}+3 p_{0}+11\right)}}\left\|V^{\varepsilon_{0}}\right\|_{L^{2}}^{q_{0} \cdot \frac{23-17 p_{0}-3 p_{0}^{2}}{2 p_{0}\left(3 p_{0}+11\right)}}\|\eta\|_{L^{p_{0}}}^{\frac{3 p_{0}^{2}+23 p_{0}-11}{2\left(3 p_{0}+11\right)}}+\left\|r u_{0}^{\theta}\right\|_{L^{\frac{1}{6 p_{0}}}}^{\frac{2}{3\left(p_{0}-1\right)^{2}}}\|\eta\|_{L^{p_{0}}}^{\frac{p_{0}-1}{4}}\right) \\
& \quad \times\left(\left\|\nabla|\eta|^{\frac{p_{0}}{2}}\right\|_{L^{2}}^{2}+\left\|\nabla\left|V^{\varepsilon_{0}}\right|^{\frac{q_{0}}{2}}\right\|_{L^{2}}^{2}+\left\|\frac{\left|V^{\varepsilon_{0}}\right|^{q_{0}}}{r^{2}}\right\|_{L^{1}}\right) \\
& +\left\|r u_{0}^{\theta}\right\|_{L^{\infty}}^{\frac{1}{6}}\left(\left\|\partial_{z}|W|^{\frac{11}{12}}\right\|_{L^{2}}^{2}+\left\|\frac{|W|^{\frac{11}{6}}}{r^{2}}\right\| \|_{L^{1}}\right)+\left\|r u_{0}^{\theta}\right\|_{L^{\frac{11}{30}}}^{\frac{11 p_{0}}{30\left(p_{0}-1\right)}}\left\|\left.W\right|^{\frac{11}{12}}\right\|_{L^{2}}^{\frac{3}{p_{0}}-\frac{43}{20}}\|\eta\|_{L^{p_{0}}}^{\frac{7 p_{0}}{8}-\frac{1}{2}} \\
& \times\left(\left\|\nabla|\eta|^{\frac{p_{0}}{2}}\right\|_{L^{2}}^{2}+\left\|\nabla|W|^{\frac{11}{12}}\right\|_{L^{2}}^{2}+\int_{\mathbb{R}^{3}} \frac{|W|^{\frac{11}{6}}}{r^{2}} d x\right) .
\end{aligned}
$$

Then under the smallness conditions (5.6), and

$$
\left\|r u_{0}^{\theta}\right\|_{L^{\infty}}^{\frac{1}{6}} \leq c_{0}, \quad\left\|r u_{0}^{\theta}\right\|_{L^{\frac{11}{30}}}^{\frac{11 p_{0}}{30\left(p_{0}-1\right)}}\left\|\left.W\right|^{\frac{11}{12}}\left(t_{0}\right)\right\|_{L^{2}}^{\frac{3}{p_{0}}-\frac{43}{20}}\left\|\eta\left(t_{0}\right)\right\|_{L^{p_{0}}}^{\frac{7 p_{0}}{8}-\frac{1}{2}} \leq c_{0}\left(p_{0}-1\right)
$$

we get, by a standard continued argument, as we did in the last step of the proof of Proposition 4.1, that

$$
\begin{aligned}
& \|\eta(t)\|_{L^{1}}+\|W(t)\|_{L^{\frac{11}{6}}}^{\frac{11}{6}}+\left\|V^{\varepsilon_{0}}(t)\right\|_{L^{q_{0}}}^{q_{0}}+\|\eta(t)\|_{L^{p_{0}}}^{p_{0}} \\
& \quad+\left(p_{0}-1\right)\left\|\nabla|\eta|^{\frac{p_{0}}{2}}\right\|_{L^{2}}^{2}+\int_{t_{0}}^{t} \int_{\mathbb{R}^{3}} \frac{\left|W\left(t^{\prime}\right)\right|^{\frac{11}{6}}}{r^{2}} d x d t^{\prime} \\
& \leq 2\left(\left\|\eta\left(t_{0}\right)\right\|_{L^{1}}+\left\|W\left(t_{0}\right)\right\|_{L^{\frac{11}{6}}}^{\frac{11}{6}}+\left\|V^{\varepsilon_{0}}\left(t_{0}\right)\right\|_{L^{q_{0}}}^{q_{0}}+\left\|\eta\left(t_{0}\right)\right\|_{L^{p_{0}}}^{p_{0}}\right) \\
& \leq \frac{C}{t^{\frac{3\left(p_{0}-1\right)}{2}}}\left(\left\|r u_{0}^{\theta}\right\|_{L^{\infty}}^{\frac{11}{6}}+L_{1, \frac{20}{3}}^{5 p_{0}}+M_{2}^{3 p_{0}}+N_{\frac{20}{13}, 20}^{5 p_{0}}\right), \quad \forall t_{0} \leq t<\infty .
\end{aligned}
$$

Recalling that $1<p_{0}<\min \left(1+\frac{1}{10 A}, \frac{21}{20}\right)$, we have $\frac{11 p_{0}}{30\left(p_{0}-1\right)}>\frac{11}{3} A$. Then it follows from Lemmas 2.1 and 5.1 that the condition (5.17) is verified provided that

$$
\left\|r u_{0}^{\theta}\right\|_{L^{\infty}} \leq \frac{c_{0}}{L_{1, \frac{20}{3}}^{6}+M_{2}^{2}+N_{\frac{20}{13}, 20}^{6}}\left(\left(p_{0}-1\right)\left(t_{0}^{\frac{21}{16} p_{0}-\frac{3}{4}}\left\|r u_{0}^{\theta}\right\|_{L^{A}}^{-A}\right)^{\frac{\left(p_{0}-1\right)}{p_{0}}}\right)^{\frac{240 p_{0}}{300-127 p_{0}-240 A\left(p_{0}-1\right)}}
$$

Finally we derive the $L^{\frac{3}{2}}$ estimate for $U$. Indeed along the same line of the derivation of $\|W(t)\|_{L^{\frac{11}{6}}}$, and using the indices given by (5.10), we infer

$$
\begin{aligned}
\frac{d}{d t}\|U(t)\|_{L^{\frac{3}{2}}}^{\frac{3}{2}}+\left\|\nabla|U|^{\frac{3}{4}}\right\|_{L^{2}}^{2} & \lesssim \int_{\mathbb{R}^{3}}\left|\frac{u^{r}}{r}\right| \cdot|U|^{\frac{3}{2}} d x \\
& \lesssim\|\eta\|_{L^{p_{0}}}^{\frac{7 p_{0}}{8}-\frac{1}{2}}\left\|\nabla|\eta|^{\frac{p_{0}}{2}}\right\|_{L^{2}}^{2\left(\frac{3}{2 p_{0}}-\frac{7}{8}\right)}\left\||U|^{\frac{3}{2}}\right\|_{L^{\frac{4}{3}}}
\end{aligned}
$$


whereas by applying Hölder's inequality, one has

$$
\begin{aligned}
\left\||U|^{\frac{3}{2}}\right\|_{L^{\frac{4}{3}}} & =\left(\int_{\mathbb{R}^{3}}|U|\left(\frac{|W|^{\frac{11}{6}}}{r^{2}}\right)^{\frac{2}{5}}\left|r^{2} U\right|^{\frac{4}{15}} d x\right)^{\frac{3}{4}} \\
& \lesssim\left\|r u^{\theta}\right\|_{L^{\frac{1}{5}} \frac{p_{0}}{5\left(p_{0}-1\right)}}\left\||U|^{\frac{3}{4}}\right\|_{L^{\frac{20 p_{0}}{20-11 p_{0}}}}\left(\int_{\mathbb{R}^{3}} \frac{|W|^{\frac{11}{6}}}{r^{2}} d x\right)^{\frac{3}{10}}, \\
& \lesssim\left\|r u^{\theta}\right\|_{L^{\frac{1}{5}} \frac{p_{0}}{5\left(p_{0}-1\right)}}\left\||U|^{\frac{3}{4}}\right\|_{L^{2}}^{\frac{3}{p_{0}}-\frac{43}{20}}\left\|\nabla|U|^{\frac{3}{4}}\right\|_{L^{2}}^{\frac{63}{20}-\frac{3}{p_{0}}}\left(\int_{\mathbb{R}^{3}} \frac{|W|^{\frac{11}{6}}}{r^{2}} d x\right)^{\frac{3}{10}} .
\end{aligned}
$$

Substituting (5.21) into (5.20), then using of Young's inequality gives rise to

$$
\begin{aligned}
\frac{d}{d t}\|U(t)\|_{L^{\frac{3}{2}}}^{\frac{3}{2}}+\left\|\nabla|U|^{\frac{3}{4}}\right\|_{L^{2}}^{2} \lesssim & \|\eta\|_{L^{p_{0}}}^{\frac{7 p_{0}}{8}-\frac{1}{2}}\left\|r u_{0}^{\theta}\right\|_{L^{\frac{1}{5\left(p_{0}-1\right)}}}^{\frac{1}{5}}\|U\|_{L^{\frac{3}{2}}}^{\frac{3}{2}}\left(\frac{3}{2 p_{0}}-\frac{43}{40}\right) \\
& \times\left(\left\|\nabla|\eta|^{\frac{p_{0}}{2}}\right\|_{L^{2}}^{2}+\left\|\nabla|U|^{\frac{3}{4}}\right\|_{L^{2}}^{2}+\int_{\mathbb{R}^{3}} \frac{|W|^{\frac{11}{6}}}{r^{2}} d x\right),
\end{aligned}
$$

from which and (5.18), we deduce by a standard continued argument that

$$
\begin{aligned}
& \|U(t)\|_{L^{\frac{3}{2}}}^{\frac{3}{2}}+\int_{t_{0}}^{t}\left\|\nabla|U|^{\frac{3}{4}}\left(t^{\prime}\right)\right\|_{L^{2}}^{2} d t^{\prime} \\
& \quad \leq 2\left\|U_{0}\right\|_{L^{\frac{3}{2}}}^{\frac{3}{2}}+\frac{C}{t^{\frac{3\left(p_{0}-1\right)}{2}}}\left(\left\|r u_{0}^{\theta}\right\|_{L^{\infty}}^{\frac{11}{6}}+L_{1, \frac{20}{3}}^{5 p_{0}}+M_{2}^{3 p_{0}}+N_{\frac{20}{13}, 20}^{5 p_{0}}\right), \quad \forall t_{0} \leq t<\infty
\end{aligned}
$$

provided that the smallness conditions (5.6), (5.17), (5.19) and

$$
\left\|\eta\left(t_{0}\right)\right\|_{L^{p_{0}}}^{\frac{7 p_{0}}{8}-\frac{1}{2}}\left\|r u_{0}^{\theta}\right\|_{L^{\frac{1}{5}} \frac{p_{0}}{5\left(p_{0}-1\right)}}\left\|U\left(t_{0}\right)\right\|_{L^{\frac{3}{2}}}^{\frac{3}{2}\left(\frac{3}{2 p_{0}}-\frac{43}{40}\right)} \leq c_{0}\left(p_{0}-1\right)
$$

hold. Yet it follows Lemma 5.1 that (5.23) can be satisfied as long as

$$
\left\|r u_{0}^{\theta}\right\|_{L^{\infty}} \leq \frac{c_{0}}{L_{1, \frac{20}{3}}^{20}+M_{2}^{12}+N_{\frac{20}{13}, 20}^{20}}\left(\left(p_{0}-1\right) \cdot\left(t_{0}^{\frac{21}{16} p_{0}-\frac{3}{4}}\left\|r u_{0}^{\theta}\right\|_{L^{A}}^{-A}\right)^{\frac{\left(p_{0}-1\right)}{p_{0}}}\right)^{\frac{5 p_{0}}{p_{0}-5 A\left(p_{0}-1\right)}} .
$$

Therefore under the smallness conditions (5.6), (5.17), (5.19) and (5.24), we get, by summing up (5.18) and (5.22) that for any $0 \leq t<\infty$,

$$
\|\eta(t)\|_{L^{1}}+\|U(t)\|_{L^{\frac{3}{2}}}^{\frac{3}{2}} \leq 2\left\|U_{0}\right\|_{L^{\frac{3}{2}}}^{\frac{3}{2}}+\frac{C}{t^{\frac{3\left(p_{0}-1\right)}{2}}}\left(\left\|r u_{0}^{\theta}\right\|_{L^{\infty}}^{\frac{11}{6}}+L_{1, \frac{20}{3}}^{5 p_{0}}+M_{2}^{3 p_{0}}+N_{\frac{20}{13}, 20}^{5 p_{0}}\right) .
$$

This completes the proof of Theorem 1.2

Acknowledgments. P. Zhang is partially supported by NSF of China under Grant 11371347 and innovation grant from National Center for Mathematics and Interdisciplinary Sciences.

\section{REFERENCES}

[1] H. Bahouri, J.-Y. Chemin and R. Danchin, Fourier Analysis and Nonlinear Partial Differential Equations, Grundlehren der Mathematischen Wissenschaften, Springer, 2010.

[2] D. Chae and J. Lee, On the regularity of the axisymmetric solutions of the Navier-Stokes equations, Math. Z., 239 (2002), 645-671.

[3] J.-Y. Chemin and P. Zhang, On the critical one compoenent regularity for 3-D Navier-Stokes system, Ann. Sci. École Norm. Sup. (4), 49 (2016), 133-169.

[4] H. Feng and V. S̆ verák, On the Cauchy problem for axi-symmetric vortex rings, Arch. Ration. Mech. Anal., 215 (2015), 89-123. 
[5] T. Gallay and V. S̆ verák, Remarks on the Cauchy problem for the axisymmetric Navier-Stokes equations, arXiv: $1510.01036 \mathrm{v} 1$

[6] T. Kato, Strong $L^{p}$-solutions of the Navier-Stokes equation in $\mathbb{R}^{m}$, with applications to weak solutions, Math. Z., 187 (1984), pages 471-480.

[7] O. A. Ladyženskaja, Unique global solvability of the three-dimensional Cauchy problem for the NavierStokes equations in the presence of axial symmetry, (Russian) Zap. Naučn. Sem. Leningrad. Otdel. Mat. Inst. Steklov. (LOMI), 7 (1968), 155-177.

[8] S. Leonardi, J. Málek, J. Něcas and M. Pokorny, On axially symmetric flows in $\mathbb{R}^{3}$, Z. Anal. Anwendungen, 18 (1999), 639-649.

[9] G. Koch, N. Nadirashvili, G. Seregin and V. Sverák, Liouville theorems for the Navier-Stokes equations and applications, Acta Math., 203 (2009), pages 83-105.

[10] J. Leray, Jean Sur le mouvement d'un liquide visqueux emplissant l'espace, Acta Math., 63 (1934), $193-248$

[11] M. R. Ukhovskii, and V. I. Iudovich, Axially symmetric flows of ideal and viscous fluids filling the whole space, J. Appl. Math. Mech., 32 (1968) 52-61.

[12] P. Zhang and T. Zhang, Global axi-symmetric solutions to 3-D Navier-Stokes System, Int. Math. Res. Not. IMRN, Vol. 2013, No. 3, 610-642.

(Y. Liu) DEPARTMENT OF MATHEMATICAL SCIENCES, UNIVERSITY OF SCIENCE AND TECHNOLOGY OF CHINA, hefei 230026, china, and Academy of Mathematics \& Systems Science, Chinese Academy of SciENCES, BEIJING 100190, CHINA.

E-mail address: liuyanlin3.14@126.com

(P. Zhang) Academy of Mathematics \& Systems Science and Hua Loo-Keng Key Laboratory of Mathematics, Chinese Academy of Sciences, Beijing 100190, CHina, and School of MathematiCal Sciences, University of Chinese Academy of Sciences, Beijing 100049, China.

E-mail address: zp@amss.ac.cn 